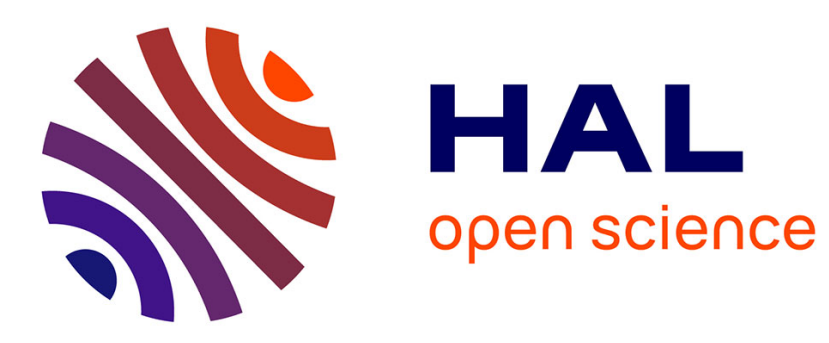

\title{
The expected number of 3D visibility events is linear
}

Olivier Devillers, Vida Dujmovic, Hazel Everett, Xavier Goaoc, Sylvain

Lazard, Hyeon-Suk Na, Sylvain Petitjean

\section{To cite this version:}

Olivier Devillers, Vida Dujmovic, Hazel Everett, Xavier Goaoc, Sylvain Lazard, et al.. The expected number of 3D visibility events is linear. SIAM Journal on Computing, 2003, 32 (6), pp.1586-1620. $10.1137 /$ S0097539702419662 . inria-00099810

\section{HAL Id: inria-00099810 https://hal.inria.fr/inria-00099810}

Submitted on 15 Dec 2009

HAL is a multi-disciplinary open access archive for the deposit and dissemination of scientific research documents, whether they are published or not. The documents may come from teaching and research institutions in France or abroad, or from public or private research centers.
L'archive ouverte pluridisciplinaire HAL, est destinée au dépôt et à la diffusion de documents scientifiques de niveau recherche, publiés ou non, émanant des établissements d'enseignement et de recherche français ou étrangers, des laboratoires publics ou privés. 


\title{
THE EXPECTED NUMBER OF 3D VISIBILITY EVENTS IS LINEAR
}

\author{
OLIVIER DEVILLERS ${ }^{\dagger}$, VIDA DUJMOVIĆ $^{\ddagger}$, HAZEL EVERETT $^{\S}$, XAVIER GOAOC $^{\S}$, \\ SYLVAIN LAZARD ${ }^{\S}$, HYEON-SUK NA ${ }^{\Uparrow}$, AND SYLVAIN PETITJEAN ${ }^{\S}$
}

\begin{abstract}
In this paper, we show that, amongst $n$ uniformly distributed unit balls in $\mathbb{R}^{3}$, the expected number of maximal non-occluded line segments tangent to four balls is linear. Using our techniques we show a linear bound on the expected size of the visibility complex, a data structure encoding the visibility information of a scene, providing evidence that the storage requirement for this data structure is not necessarily prohibitive. These results significantly improve the best previously known bounds of $O\left(n^{8 / 3}\right)[11]$.

Our results generalize in various directions. We show that the linear bound on the expected number of maximal non-occluded line segments that are not too close to the boundary of the scene and tangent to four unit balls extends to balls of various but bounded radii, to polyhedra of bounded aspect ratio, and even to non-fat 3D objects such as polygons of bounded aspect ratio. We also prove that our results extend to other distributions such as the Poisson distribution. Finally, we indicate how our probabilistic analysis provides new insight on the expected size of other global visibility data structures, notably the aspect graph.
\end{abstract}

Key words. computational geometry, $3 \mathrm{D}$ visibility, visual events, visibility complex, probabilistic analysis, expected complexity.

AMS subject classifications. 68U05, 60D05

1. Introduction. Visibility computations are central in computer graphics applications. Computing the limits of the umbra and penumbra cast by an area light source, identifying the set of blockers between any two polygons and determining the view from a given point are examples of visibility queries that are essential for the realistic rendering of 3D scenes. In global illumination algorithms, where the flow of light in a scene is simulated according to the laws of geometrical optics, visibility computations are excessively costly. In fact, more than half of the overall computation time can routinely be spent on visibility queries in radiosity simulations [13].

One approach to speeding up rendering is to store global visibility information in a data structure which can then be efficiently queried. The visibility complex, a partition of the set of maximal free line segments, has been proposed as a unified data structure encoding the visibility information of a scene [23] and has been used for rendering purposes [11]. Other related data structures include Pellegrini's rayshooting structure [20], the aspect graph [22] and the visual hull [14]; see [8] for a recent survey.

One problem with these types of data structures which may prevent their application in practice is their potentially enormous size; the size of the visibility complex of a set of $n$ triangles in $\mathbb{R}^{3}$ is $\Theta\left(n^{4}\right)$ in the worst case [11], which is prohibitive even for scenes of relatively modest size. Worst-case examples are somewhat artificial and indeed Durand, Drettakis and Puech [9] provide empirical evidence indicating that these

$\dagger$ INRIA Sophia-Antipolis. Olivier.Devillers@inria.fr. http://www-sop.inria.fr/prisme/. Partially supported by the IST Programme of the EU as a Shared-cost RTD (FET Open) Project under Contract No IST-2000-26473 (ECG - Effective Computational Geometry for Curves and Surfaces).

$\ddagger$ School of Computer Science, McGill University; vida@cs.mcgill.ca. Research supported by FCAR.

$\S$ LORIA - INRIA Lorraine, CNRS, Univ. Nancy 2. \{everett, goaoc, lazard, petitjea\}@loria.fr. http://www.loria.fr/ $\sim$ \{everett, goaoc, lazard, petitjea $\} /$. Research supported by the McGill-ISA collaborative INRIA project.

I School of Computing, Soongsil University, Seoul, South Korea. hsnaa@computing.ssu.ac.kr. This author's research was done during a post-doctoral tenure at LORIA - INRIA Lorraine. 
worst-case upper bounds are largely pessimistic in practical situations; they observe a quadratic growth rate, albeit for rather small scenes. In $2 \mathrm{D}$, while the worst-case complexity of the visibility complex is quadratic, experimental results strongly suggest that the size of the visibility complex of a scene consisting of scattered triangles is linear [4].

Our goal is to provide theoretical evidence to support these observations. To this end we investigate the expected size of the visibility complex, or equivalently, the expected number of visibility events, occurring in scenes in $\mathbb{R}^{3}$. A visibility event is a combinatorial change in the view of a moving observer; such an event occurs when the viewing direction becomes tangent to some objects. For sets of convex objects in general position in $\mathbb{R}^{3}$, the viewing direction can be tangent to at most four objects. Visibility events thus correspond to maximal non-occluded line segments tangent to at most four objects; combinatorially different visibility events correspond to the faces of the visibility complex.

In this paper we prove that the expected number of maximal non-occluded line segments tangent to four balls, amongst $n$ uniformly distributed unit balls in $\mathbb{R}^{3}$, is linear. This improves the previously known upper bound of $O\left(n^{8 / 3}\right)$ by Durand et al. who proved the more general result that the expected number of (possibly occluded) lines tangent to four balls is $O\left(n^{8 / 3}\right)$ for the same model [11]. The intuition behind our proof is that, given a line segment tangent to four balls, the probability that this segment is not occluded by any other ball is the probability that a cylinder-like volume of radius 1 about the segment is free from the centers of the other balls. This probability decays roughly exponentially fast with the length of the segment, yielding the result. Using our techniques we then show a linear bound on the expected size of the visibility complex of $n$ uniformly distributed unit balls in $\mathbb{R}^{3}$. A simple computation then provides us with the same result for the Poisson distribution.

Our results generalize in the following ways. We show that, for certain types of visibility events, the linear bound also applies to balls of various but bounded radii, to polyhedral objects enclosed between two concentric balls of fixed radius, and even to non-fat objects such as polygons, enclosed between two concentric circles of fixed radius, whose centers and normals are uniformly distributed. For the remaining types of visibility events (namely those occurring close to the boundary of the scene - see Section 7.3 for the details), we prove only an $O\left(n^{2}\right)$ bound, which is still an improvement over the bound by Durand et al. [11].

Of course objects in graphics scenes are seldom distributed uniformly or according to a Poisson point process. We chose this model because it allows tractable proofs of theoretical results. This is important in a context where there are few rigorous results either theoretical or experimental. The same model, albeit with significant simplifying assumptions, has also been used to study the average complexity of ray shooting [25, 26] and occlusion culling for 2D urban scenes [18]. It is interesting to note that Szirmay-Kalos et al. [25], after establishing bounds on the average complexity of ray shooting in scenes consisting of unit balls distributed according to a Poisson point process, tested their algorithms on a small number of realistic scenes. The results they obtain are consistent with those predicted by the theoretical results thus providing some evidence that the model is helpful. No other model has been widely accepted by the graphics community and, in fact, generating meaningful random scenes usable for testing algorithms is a major problem. (Note that rather than attempting to generate random scenes, an alternative approach, which has been used to study the average complexity of ray shooting, is to fix the scene and randomly distribute the rays; see, 


\begin{tabular}{|c|c|c|}
\hline & Worst-case & Expected \\
\hline \hline possibly occluded lines amongst unit balls & $\Theta\left(n^{4}\right)$ & $O\left(n^{\frac{8}{3}}\right)[11]$ \\
\hline free lines amongst unit balls & $\Omega\left(n^{2}\right)[\star], O\left(n^{3+\epsilon}\right)[1]$ & $\Theta(n)[\star]$ \\
\hline free lines amongst disjoint homothetic polytopes & $\Omega\left(n^{3}\right)[3]$ & $?$ \\
\hline free segments amongst unit balls & $\Omega\left(n^{2}\right)[\star], O\left(n^{4}\right)$ & $\Theta(n)[\star]$ \\
\hline free segments amongst arbitrary sized balls & $\Omega\left(n^{3}\right)[6], O\left(n^{4}\right)$ & $?$ \\
\hline visibility complex of unit balls & $\Omega\left(n^{2}\right)[\star], O\left(n^{4}\right)$ & $\Theta(n)[\star]$ \\
\hline
\end{tabular}

Table 1.1. Known bounds on the complexity of the set of lines, free lines or maximal free line segments tangent to 4 amongst $n$ objects. The expected complexities are calculated for the uniform distribution. The results referenced by $\star$ are established in this paper.

for example, [2].)

Previous results on this topic include those that bound the number of lines and the number of free (i.e., non-occluded) lines amongst different sets of objects. They are summarized in Table 1.1. Agarwal, Aronov and Sharir [1] showed an upper bound of $O\left(n^{3+\epsilon}\right)$ on the complexity of the space of line transversals of $n$ balls by studying the lower envelope of a set of functions. A study of the upper envelope of the same set of functions yields the same upper bound on the number of free lines tangent to four balls [6]. Agarwal et al. [1] also showed a lower bound on the complexity of the space of line transversals of $n$ balls of $\Omega\left(n^{3}\right)$ for arbitrarily sized balls and $\Omega\left(n^{2}\right)$ for unit sized balls. De Berg, Everett and Guibas [3] showed a $\Omega\left(n^{3}\right)$ lower bound on the number of free lines (and thus free segments) tangent to four amongst $n$ disjoint homothetic convex polyhedra. Recently, Devillers and Ramos [6] presented a simple $\Omega\left(n^{3}\right)$ lower bound on the number of free segments tangent to 4 amongst $n$ arbitrarily sized balls, which also holds for non-intersecting balls. We also present a simple $\Omega\left(n^{2}\right)$ lower bound on the number of free segments tangent to 4 amongst $n$ unit balls.

In the next section we carefully define the problem and state our main results. In Section 3 and Section 4 we prove the expected upper and lower linear bounds on the number of free segments tangent to four balls. In Section 5 we extend this result to the visibility complex. We present in Section 6 a $\Omega\left(n^{2}\right)$ worst-case lower bound. In Section 7 we discuss extensions of our results to some other models. We conclude in Section 8 .

2. Our model and results. We first describe our objects and their distribution. Let $n \in \mathbb{N}$ and $\mu$ be a positive constant. A sample scene consists of $n$ unit radius balls $B_{1}, \ldots, B_{n}$ whose centers $p_{1}, \ldots, p_{n}$ are independently chosen from the uniform distribution over a universal ball $\mathcal{U}$ of radius $R$ centered at $O$. Since we distribute the centers $p_{i}$ over $\mathcal{U}$, the balls $B_{i}$ may intersect each other and are contained in the ball, denoted $\mathcal{U}^{+}$, whose radius is $R+1$ and whose center is that of $\mathcal{U}$.

We define the radius $R$ of the universal ball $\mathcal{U}$ to be a function of $n$ satisfying

$$
R^{3}=n / \mu \text {. }
$$

The constant $\mu$ reflects the density of the balls in the sense that the expected number of centers lying in any given solid of volume $V$ in the universe is $\frac{3}{4 \pi} \mu V$. (The model is interesting only if $n$ is asymptotically proportional to $R^{3}$. Indeed, if $\frac{n}{R^{3}}$ tends to infinity when $n$ tends to infinity, then the universe gets entirely filled up with balls and visibility events only occur in $\mathcal{U}^{+} \backslash \mathcal{U}$. Conversely, if $\frac{n}{R^{3}}$ tends to zero when $n$ tends to infinity, then the balls get scattered so far apart that the probability that any four (or three) balls have a common tangent goes to zero.) 
We now define the visibility complex of a set of objects [23]. A free or non-occluded segment is a line segment that does not intersect the interior of any object. A free segment is maximal if it is not properly contained in another one. Thus, the endpoints of a maximal free segment are either on an object or at infinity. We say that two maximal free segments are similar if their endpoints lie on the same objects (possibly at infinity). The visibility complex of a collection of objects is roughly defined as the partition of the space of maximal free segments into connected components of similar segments ${ }^{1}$. Its faces have dimension between 0 and 4 ; when the objects are in adequate general position, a $k$-dimensional face corresponds to a connected set of similar maximal non-occluded line segments tangent to $4-k$ objects.

In order to bound the total number of faces of the visibility complex, we first bound the number of 0 -faces. To do this, we count the T4-segments, which are the free segments tangent to 4 balls with endpoints on two of those balls. Since there is a one-to-one correspondence between 0 -faces and $T 4$-segments when the objects are in adequate general position, this yields a bound on the expected number of vertices of the visibility complex. Note that since the balls are contained in $\mathcal{U}^{+}$, the $T 4$-segments are also contained in $\mathcal{U}^{+}$.

Our main result is the following.

THEOREM 2.1. The expected number of T4-segments amongst $n$ uniformly distributed unit balls is $\Theta(n)$.

We extend this result to the higher dimensional faces of the complex.

THEOREM 2.2. The expected size of the visibility complex of $n$ uniformly distributed unit balls is $\Theta(n)$.

We also present an $\Omega\left(n^{2}\right)$ worst-case lower bound on the number of $T 4$-segments amongst $n$ unit balls in $\mathbb{R}^{3}$ (see Proposition 6.1). In fact the lower bound holds for the number of $k$-faces of the visibility complex, for all $k$ between 0 and 4 .

3. The expected number of T4-segments is at most linear. The general idea behind the proof of the upper bound of Theorem 2.1 is the following. For any ordered choice of four balls, we bound from above the probability that a line is tangent to these balls in the given order and is not occluded in between its contact points with the balls. Then we sum these probabilities over all ordered quadruples of balls and all potential tangent lines to these balls.

For any two points $p$ and $q$, and positive real number $\alpha$, let $\mathcal{H}(p, q, \alpha)$ denote the union of all the balls of radius $\alpha$ centered on the line segment $p q$ (see Figure 3.1). We first show that a line is tangent to four balls $B_{i}, B_{j}, B_{k}$ and $B_{l}$ in that order only if $p_{j}$ and $p_{k}$ are in $\mathcal{H}\left(p_{i}, p_{l}, 2\right)$. Thus the volume of $\mathcal{H}\left(p_{i}, p_{l}, 2\right) \cap \mathcal{U}$ gives an upper bound on the probability that a line tangent to the four balls, in the given order, exists.

We next show that a segment tangent to four balls $B_{i}, B_{j}, B_{k}$ and $B_{l}$ in that order, at points $t_{i}, t_{j}, t_{k}$ and $t_{l}$, respectively, is not occluded if and only if the centers of all remaining balls are outside or on the boundary of $\mathcal{H}\left(t_{i}, t_{l}, 1\right)$. The volume of $\mathcal{U} \backslash \mathcal{H}\left(t_{i}, t_{l}, 1\right)$ gives an upper bound on the probability that the tangent segment is not occluded. Thus, to get an upper bound on that probability, we need a lower bound on the volume of $\mathcal{H}\left(t_{i}, t_{l}, 1\right) \cap \mathcal{U}$.

To bound the probability that a $T 4$-segment exists, we integrate over the distance between $p_{i}$ and $p_{l}$, and over the distance from $p_{i}$ to the boundary of the universe $\mathcal{U}$.

\footnotetext{
${ }^{1}$ Formally, we consider the space of free segments quotiented by the equivalence relation that is the transitive and reflexive closure of the inclusion. In other words, two free segments are identified if they are both contained in the same maximal free segment. This allows the cells of the partition to be connected.
} 
This integral is split into three parts covering the cases where

(i) $B_{i}$ and $B_{l}$ are close to one another,

(ii) at least one of $B_{i}$ and $B_{l}$ is entirely inside the universe,

(iii) $B_{i}$ and $B_{l}$ are not close to one another and both are partially outside the universe.

In each case we over-estimate the volume of $\mathcal{H}\left(p_{i}, p_{l}, 2\right) \cap \mathcal{U}$ and under-estimate the volume of $\mathcal{H}\left(t_{i}, t_{l}, 1\right) \cap \mathcal{U}$. We apply the same general proof technique in each of the three cases. While Case (ii) illustrates the main idea behind the proof (Case (i) being a simplified version), extending this idea to Case (iii) is technically challenging because of the difficulties caused by the boundary of the universe.

3.1. Definitions. Let $\mathcal{N}$ be the set of ordered 4 -tuples $(i, j, k, l)$ chosen from $\{1,2, \ldots, n\}$ such that $i, j, k, l$ are pairwise distinct. In our model, the probability that four centers are collinear is zero, so we may assume that any set of four balls admits at most 12 real common tangent lines [5, 15]. Moreover, the real common tangent lines correspond to the real solutions of a degree 12 system of equations. For any set of four balls we order arbitrarily the 12 solutions of the associated system.

Given four balls $B_{i}, B_{j}, B_{k}$ and $B_{l}$, we denote by $\mathcal{L}_{i, j, k, l}^{\omega}$, for $\omega$ in $\{1, \ldots, 12\}$, the event that the $\omega^{\text {th }}$ solution of the system is real, that the corresponding real tangent line is tangent to the four balls $B_{i}, B_{j}, B_{k}$ and $B_{l}$ in that order, and that $p_{i}$ is not closer than $p_{l}$ to the boundary of $\mathcal{U}$. Whenever $\mathcal{L}_{i, j, k, l}^{\omega}$ occurs, we denote the points of tangency of that line on $B_{i}, B_{j}, B_{k}, B_{l}$ by $t_{i}, t_{j}, t_{k}, t_{l}$, respectively. Let $\delta_{i, j, k, l}^{\omega}$ be the event that $\mathcal{L}_{i, j, k, l}^{\omega}$ occurs and the line segment $t_{i} t_{l}$ is not occluded. Notice that if $\delta_{i, j, k, l}^{\omega}$ occurs, the balls $B_{i}, B_{j}, B_{k}, B_{l}$ define a $T 4$-segment, and that a $T 4$-segment corresponds to a unique $\delta_{i, j, k, l}^{\omega}$.

Let $\mathbf{x}_{i, l}$ be the random variable representing the distance from $p_{i}$ to $p_{l}$, and $\mathbf{y}_{i}$ (resp. $\mathbf{y}_{l}$ ) be the random variable denoting the distance from $p_{i}$ (resp. $p_{l}$ ) to the boundary of the universe.

In the sequel, a random point $p$ denotes a point chosen from the uniform distribution over $\mathcal{U}$.

3.2. The Proof. There is a one-to-one correspondence between the $T 4$-segments and the events $\delta_{i, j, k, l}^{\omega}$ that occur. We thus have the following straightforward lemma.

LEMмA 3.1. The expected number of T4-segments amongst $n$ uniformly distributed unit balls is

$$
\sum_{(i, j, k, l) \in \mathcal{N}} \sum_{\omega=1}^{12} \operatorname{Pr}\left(\delta_{i, j, k, l}^{\omega}\right) .
$$

We bound the probability $\operatorname{Pr}\left(\delta_{i, j, k, l}^{\omega}\right)$ by integrating over the distance $x$ between $p_{i}$ and $p_{l}$, and over the distance $y$ from $p_{i}$ to the boundary of the universe $\mathcal{U}$. The integral is split into three parts covering the cases where (i) the balls $B_{i}$ and $B_{l}$ are close to one another, (ii) $p_{i}$ is at distance at least 1 from the boundary of $\mathcal{U}$, and (iii) the balls $B_{i}$ and $B_{l}$ are not close to one another and $p_{i}$ is at distance less than 1 from the boundary of $\mathcal{U}$. Note that in the last case, if $\delta_{i, j, k, l}^{\omega}$ occurs, then both ball centers $p_{i}$ and $p_{l}$ are within distance 1 from the boundary of $\mathcal{U}$. Two balls are considered close to one another if their centers are closer than some sufficiently large constant; for technical reasons which are embedded in the proof of Proposition A.1, we actually define close to mean distance at most 6 . 


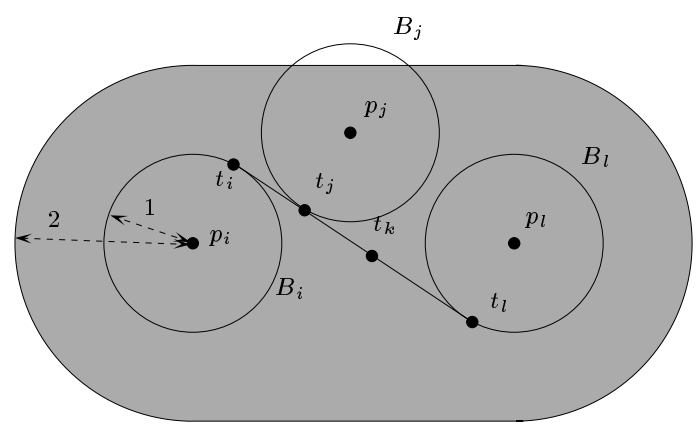

(a) $\mathcal{H}\left(p_{i}, p_{l}, 2\right)$

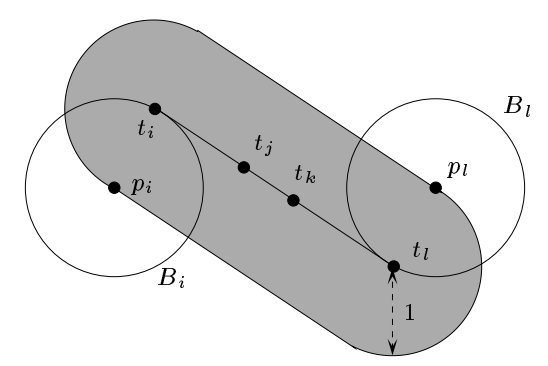

(b) $\mathcal{H}\left(t_{i}, t_{l}, 1\right)$

Fig. 3.1. $\mathcal{H}\left(p_{i}, p_{l}, 2\right)$ and $\mathcal{H}\left(t_{i}, t_{l}, 1\right)$ are shown shaded.

Lemma 3.2. $\operatorname{Pr}\left(\delta_{i, j, k, l}^{\omega}\right) \leqslant I_{x \leqslant 6}+I_{y \geqslant 1}+I_{x>6, y<1}$, where

$$
\begin{gathered}
I_{x \leqslant 6}=\int_{x=0}^{6} \operatorname{Pr}\left(\delta_{i, j, k, l}^{\omega} \mid \mathbf{x}_{i, l}=x\right) \cdot \operatorname{Pr}\left(x \leqslant \mathbf{x}_{i, l}<x+d x\right), \\
I_{y \geqslant 1}=\int_{x=0}^{2 R} \operatorname{Pr}\left(\delta_{i, j, k, l}^{\omega} \mid \mathbf{x}_{i, l}=x, \mathbf{y}_{i} \geqslant 1\right) \cdot \operatorname{Pr}\left(x \leqslant \mathbf{x}_{i, l}<x+d x \mid \mathbf{y}_{i} \geqslant 1\right), \\
I_{x>6, y<1}=\int_{x=6}^{2 R} \int_{y=0}^{1} \operatorname{Pr}\left(\delta_{i, j, k, l}^{\omega} \mid \mathbf{x}_{i, l}=x, \mathbf{y}_{i}=y, \mathbf{y}_{l} \leqslant \mathbf{y}_{i}\right) \\
\cdot \operatorname{Pr}\left(\left(x \leqslant \mathbf{x}_{i, l}<x+d x\right) \cap\left(\mathbf{y}_{l} \leqslant \mathbf{y}_{i}\right) \mid \mathbf{y}_{i}=y\right) \\
\cdot \operatorname{Pr}\left(y \leqslant \mathbf{y}_{i}<y+d y\right) .
\end{gathered}
$$

Proof. By the Total Probability Theorem (see [19]),

$$
\operatorname{Pr}\left(\delta_{i, j, k, l}^{\omega}\right)=\int_{x=0}^{2 R} \operatorname{Pr}\left(\delta_{i, j, k, l}^{\omega} \mid \mathbf{x}_{i, l}=x\right) \cdot \operatorname{Pr}\left(x \leqslant \mathbf{x}_{i, l}<x+d x\right) .
$$

The integral can be split at $x=6$, giving $I_{x \leqslant 6}$. Then applying the Total Probability Theorem on what remains, we get

$$
\begin{gathered}
\int_{x=6}^{2 R} \int_{y=0}^{R} \operatorname{Pr}\left(\delta_{i, j, k, l}^{\omega} \mid \mathbf{x}_{i, l}=x, \mathbf{y}_{i}=y\right) \cdot \operatorname{Pr}\left(\left(x \leqslant \mathbf{x}_{i, l}<x+d x\right) \mid \mathbf{y}_{i}=y\right) \\
\cdot \operatorname{Pr}\left(y \leqslant \mathbf{y}_{i}<y+d y\right)
\end{gathered}
$$


which can be split at $y=1$. The part corresponding to $y$ between 1 and $R$ is equal to

$$
\begin{aligned}
& \int_{x=6}^{2 R} \int_{y=1}^{R} \operatorname{Pr}\left(\delta_{i, j, k, l}^{\omega} \mid \mathbf{x}_{i, l}=x, \mathbf{y}_{i}=y, \mathbf{y}_{i} \geqslant 1\right) \\
& \quad \cdot \operatorname{Pr}\left(\left(x \leqslant \mathbf{x}_{i, l}<x+d x\right) \mid \mathbf{y}_{i}=y, \mathbf{y}_{i} \geqslant 1\right) \cdot \operatorname{Pr}\left(y \leqslant \mathbf{y}_{i}<y+d y\right) \\
& \leqslant \int_{x=6}^{2 R} \int_{y=0}^{R} \operatorname{Pr}\left(\delta_{i, j, k, l}^{\omega} \cap\left(x \leqslant \mathbf{x}_{i, l}<x+d x\right) \mid \mathbf{y}_{i}=y, \mathbf{y}_{i} \geqslant 1\right) \cdot \operatorname{Pr}\left(y \leqslant \mathbf{y}_{i}<y+d y\right) .
\end{aligned}
$$

Applying the Total Probability Theorem again, we get

$$
\int_{x=6}^{2 R} \operatorname{Pr}\left(\delta_{i, j, k, l}^{\omega} \cap\left(x \leqslant \mathbf{x}_{i, l}<x+d x\right) \mid \mathbf{y}_{i} \geqslant 1\right)
$$

which is less than $I_{y \geqslant 1}$. Consider now the part of (3.1) for $y$ between 0 and 1 . If $\mathbf{y}_{l}>\mathbf{y}_{i}$ then $\delta_{i, j, k, l}^{\omega}$ does not occur (by definition of $\mathcal{L}_{i, j, k, l}^{\omega}$ ), thus we have

$$
\begin{aligned}
& \operatorname{Pr}\left(\delta_{i, j, k, l}^{\omega} \mid \mathbf{x}_{i, l}=x, \mathbf{y}_{i}=y\right) \cdot \operatorname{Pr}\left(\left(x \leqslant \mathbf{x}_{i, l}<x+d x\right) \mid \mathbf{y}_{i}=y\right) \\
&=\operatorname{Pr}\left(\delta_{i, j, k, l}^{\omega} \cap\left(x \leqslant \mathbf{x}_{i, l}<x+d x\right) \mid \mathbf{y}_{i}=y\right) \\
&=\operatorname{Pr}\left(\delta_{i, j, k, l}^{\omega} \cap\left(x \leqslant \mathbf{x}_{i, l}<x+d x\right) \cap\left(\mathbf{y}_{l} \leqslant \mathbf{y}_{i}\right) \mid \mathbf{y}_{i}=y\right) \\
&=\operatorname{Pr}\left(\delta_{i, j, k, l}^{\omega} \mid \mathbf{x}_{i, l}=x, \mathbf{y}_{i}=y, \mathbf{y}_{l} \leqslant \mathbf{y}_{i}\right) \\
& \cdot \operatorname{Pr}\left(\left(x \leqslant \mathbf{x}_{i, l}<x+d x\right) \cap\left(\mathbf{y}_{l} \leqslant \mathbf{y}_{i}\right) \mid \mathbf{y}_{i}=y\right) .
\end{aligned}
$$

Thus, the part of (3.1) for $y$ between 0 and 1 is equal to $I_{x>6, y<1}$.

Let $\Xi$ denote any of the following events: $\left(\mathbf{x}_{i, l}=x\right),\left(\mathbf{x}_{i, l}=x, \mathbf{y}_{i} \geqslant 1\right),\left(\mathbf{x}_{i, l}=\right.$ $\left.x, \mathbf{y}_{i}=y, \mathbf{y}_{l} \leqslant \mathbf{y}_{i}\right)$. The next three lemmas are used to bound $\operatorname{Pr}\left(\delta_{i, j, k, l}^{\omega} \mid \Xi\right)$ appearing in the three integrals $I_{x \leqslant 6}, I_{y \geqslant 1}$ and $I_{x>6, y<1}$.

LEMMA 3.3. If a line is tangent to four balls $B_{i}, B_{j}, B_{k}, B_{l}$ in that order at $t_{i}, t_{j}, t_{k}, t_{l}$, respectively, then $p_{j}, p_{k} \in \mathcal{H}\left(p_{i}, p_{l}, 2\right)$. Also, the segment $t_{i} t_{l}$ is not occluded if and only if the interior of $\mathcal{H}\left(t_{i}, t_{l}, 1\right)$ does not contain the center of any other ball.

Proof. Segment $t_{i} t_{l}$ is contained in $\mathcal{H}\left(p_{i}, p_{l}, 1\right)$. Since $t_{j}$ and $t_{k}$ belong to that segment, $t_{j}$ and $t_{k}$ are also in $\mathcal{H}\left(p_{i}, p_{l}, 1\right)$. Thus $p_{j}, p_{k}$ are both in $\mathcal{H}\left(p_{i}, p_{l}, 2\right)$. See Figure 3.1 (a).

The segment $t_{i} t_{l}$ is occluded if and only if some ball $B_{\gamma}, \gamma \neq i, j, k, l$, properly intersects it, that is the center of $B_{\gamma}$ lies in the interior of $\mathcal{H}\left(t_{i}, t_{l}, 1\right)$. See Figure 3.1 (b).

Lemma 3.4. $\operatorname{Pr}\left(p \in \mathcal{H}\left(p_{i}, p_{l}, 2\right) \mid \Xi\right) \leqslant \frac{(3 x+8)}{R^{3}}$.

Proof.

$\operatorname{Pr}\left(p \in \mathcal{H}\left(p_{i}, p_{l}, 2\right) \mid \Xi\right)=\left.\frac{\text { Volume of } \mathcal{H}\left(p_{i}, p_{l}, 2\right) \cap \mathcal{U}}{\text { Volume of } \mathcal{U}}\right|_{\Xi} \leqslant\left.\frac{\text { Volume of } \mathcal{H}\left(p_{i}, p_{l}, 2\right)}{\text { Volume of } \mathcal{U}}\right|_{\Xi}$

When $\Xi$ occurs, $\mathbf{x}_{i, l}=x$ and the volumes of $\mathcal{H}\left(p_{i}, p_{l}, 2\right)$ and $\mathcal{U}$ are $\frac{4 \pi}{3}(3 x+8)$ and $\frac{4 \pi}{3} R^{3}$, respectively. Thus

$$
\operatorname{Pr}\left(p \in \mathcal{H}\left(p_{i}, p_{l}, 2\right) \mid \Xi\right) \leqslant \frac{3 x+8}{R^{3}}
$$


Lemma 3.5. $\operatorname{Pr}\left(\delta_{i, j, k, l}^{\omega} \mid \Xi\right) \leqslant \frac{(3 x+8)^{2}}{R^{6}} \cdot \operatorname{Pr}\left(p \notin \mathcal{H}\left(t_{i}, t_{l}, 1\right) \mid \mathcal{L}_{i, j, k, l}^{\omega}, \Xi\right)^{n-4}$.

Proof. If $\delta_{i, j, k, l}^{\omega}$ occurs, then $\mathcal{L}_{i, j, k, l}^{\omega}$ necessarily occurs, thus

$$
\operatorname{Pr}\left(\delta_{i, j, k, l}^{\omega} \mid \Xi\right)=\operatorname{Pr}\left(\delta_{i, j, k, l}^{\omega} \cap \mathcal{L}_{i, j, k, l}^{\omega} \mid \Xi\right)=\operatorname{Pr}\left(\mathcal{L}_{i, j, k, l}^{\omega} \mid \Xi\right) \cdot \operatorname{Pr}\left(\delta_{i, j, k, l}^{\omega} \mid \mathcal{L}_{i, j, k, l}^{\omega}, \Xi\right) .
$$

By Lemma 3.3, $\operatorname{Pr}\left(\mathcal{L}_{i, j, k, l}^{\omega} \mid \Xi\right)$ is bounded by the probability that $p_{j}$ and $p_{k}$ belong to $\mathcal{H}\left(p_{i}, p_{l}, 2\right)$ given $\Xi$, and $\operatorname{Pr}\left(\delta_{i, j, k, l}^{\omega} \mid \mathcal{L}_{i, j, k, l}^{\omega}\right)$ is equal to the probability that for all $\gamma \neq i, j, k, l$, point $p_{\gamma}$ is outside $\mathcal{H}\left(t_{i}, t_{l}, 1\right)$ given $\Xi$. Since all the points are independently and identically drawn from the uniform distribution over $\mathcal{U}$, Lemma 3.4 yields the result.

We consider the three integrals $I_{x \leqslant 6}, I_{y \geqslant 1}$ and $I_{x>6, y<1}$ in the following subsections, and prove that each is bounded by $O\left(\frac{1}{n^{3}}\right)$. This will complete the proof of the upper bound of Theorem 2.1 since, by Lemmas 3.1 and 3.2, the expected number of T4-segments is less than $12\left(\begin{array}{c}n \\ 4\end{array}\right)\left(I_{x \leqslant 6}+I_{y \geqslant 1}+I_{x>6, y<1}\right)$.

3.2.1. $\mathbf{B}_{i}$ and $\mathbf{B}_{l}$ are close to one another. We prove here that $I_{x \leqslant 6}$ is $O\left(\frac{1}{n^{3}}\right)$. When $B_{i}$ and $B_{l}$ are close to one another, the probability that there exist two other balls, $B_{j}$ and $B_{k}$, defining a line tangent to $B_{i}, B_{j}, B_{k}, B_{l}$ in that order, is small enough that we do not need to consider occlusions in order to get the bound we want.

We first bound the term $\operatorname{Pr}\left(x \leqslant \mathbf{x}_{i, l}<x+d x\right)$ appearing in the integral $I_{x \leqslant 6}$.

Lemma 3.6. $\operatorname{Pr}\left(x \leqslant \mathbf{x}_{i, l}<x+d x\right) \leqslant \frac{3 x^{2}}{R^{3}} d x$.

Proof. When $p_{i}$ is given, $p_{l}$ must belong to a spherical shell between two spheres of center $p_{i}$ and radii $x$ and $x+d x$. The probability $\operatorname{Pr}\left(x \leqslant \mathbf{x}_{i, l}<x+d x\right)$, if $p_{i}$ is known, is exactly the volume of the part of the spherical shell inside $\mathcal{U}$ divided by the volume of $\mathcal{U}$. The volume of the part of the spherical shell inside $\mathcal{U}$ is bounded from above by the volume of the spherical shell which is $4 \pi x^{2} d x$. Since the volume of $\mathcal{U}$ is $\frac{4}{3} \pi R^{3}$ we get the claimed bound. (The exact value of $\operatorname{Pr}\left(x \leqslant \mathbf{x}_{i, l}<x+d x\right)$ is actually given in $[17,24]$ but the above approximate bound is enough for our purposes.)

Proposition 3.7. $I_{x \leqslant 6}$ is $O\left(\frac{1}{n^{3}}\right)$.

Proof. Recall that (see Lemma 3.2)

$$
I_{x \leqslant 6}=\int_{x=0}^{6} \operatorname{Pr}\left(\delta_{i, j, k, l}^{\omega} \mid \mathbf{x}_{i, l}=x\right) \cdot \operatorname{Pr}\left(x \leqslant \mathbf{x}_{i, l}<x+d x\right) .
$$

By Lemma 3.5,

$$
\begin{aligned}
\operatorname{Pr}\left(\delta_{i, j, k, l}^{\omega} \mid \mathbf{x}_{i, l}=x\right) & \leqslant \frac{(3 x+8)^{2}}{R^{6}} \cdot \operatorname{Pr}\left(p \notin \mathcal{H}\left(t_{i}, t_{l}, 1\right) \mid \mathbf{x}_{i, l}=x, \mathcal{L}_{i, j, k, l}^{\omega}\right)^{n-4} \\
& \leqslant \frac{(3 x+8)^{2}}{R^{6}} .
\end{aligned}
$$

It thus follows from Lemma 3.6 that

$$
I_{x \leqslant 6} \leqslant \int_{x=0}^{6} \frac{(3 x+8)^{2}}{R^{6}} \cdot \frac{3 x^{2}}{R^{3}} d x=\frac{\mu^{3}}{n^{3}} \int_{x=0}^{6} 3 x^{2}(3 x+8)^{2} d x=O\left(\frac{1}{n^{3}}\right) .
$$


3.2.2. $\mathbf{B}_{i}$ is entirely inside $\mathcal{U}$. For the integral $I_{y \geqslant 1}$, occlusions must be taken into account. To this aim, we bound from below the volume of $\mathcal{H}\left(t_{i}, t_{l}, 1\right) \cap \mathcal{U}$ in the following lemma.

LEMMA 3.8. When $\mathcal{L}_{i, j, k, l}^{\omega}$ occurs and $\mathbf{y}_{i} \geqslant 1$, the volume of $\mathcal{H}\left(t_{i}, t_{l}, 1\right) \cap \mathcal{U}$ is greater than $\frac{\pi}{12} \mathbf{x}_{i, l}$.

Proof. Let $K$ be the ball having diameter $p_{i} t_{i}$. Note that $K$ and $p_{l}$ are both contained in $\mathcal{U}$ and in $\mathcal{H}\left(t_{i}, t_{l}, 1\right)$. The convex hull of $p_{l}$ and $K$ is thus contained in $\mathcal{H}\left(t_{i}, t_{l}, 1\right) \cap \mathcal{U}$, and its volume is larger than half the volume of the ball $K, \frac{\pi}{12}$, plus the volume of a cone of apex $p_{l}$, of base a disk whose boundary is a great circle of $K$, and of height greater than $\mathbf{x}_{i, l}-1$. The volume of that cone is at least $\frac{1}{3} \frac{\pi}{2^{2}}\left(\mathbf{x}_{i, l}-1\right)=\frac{\pi}{12} \mathbf{x}_{i, l}-\frac{\pi}{12}$.

We now bound the probability that a tangent line segment $t_{i} t_{l}$ is not occluded by any of the other $n-4$ balls, given that the line segment $t_{i} t_{l}$ exists and the ball $B_{i}$ is entirely contained in $\mathcal{U}$.

LEMma 3.9. $\operatorname{Pr}\left(p \notin \mathcal{H}\left(t_{i}, t_{l}, 1\right) \mid \mathbf{x}_{i, l}=x, \mathbf{y}_{i} \geqslant 1, \mathcal{L}_{i, j, k, l}^{\omega}\right)^{n-4}<55 \exp \left(-\frac{\mu x}{16}\right)$.

Proof. First notice that

$$
\begin{aligned}
\operatorname{Pr}\left(p \notin \mathcal{H}\left(t_{i}, t_{l}, 1\right) \mid \mathbf{x}_{i, l}=\right. & \left.x, \mathbf{y}_{i} \geqslant 1, \mathcal{L}_{i, j, k, l}^{\omega}\right) \\
& =1-\left.\frac{\text { Volume of } \mathcal{H}\left(t_{i}, t_{l}, 1\right) \cap \mathcal{U}}{\text { Volume of } \mathcal{U}}\right|_{\mathbf{x}_{i, l}=x, \mathbf{y}_{i} \geqslant 1, \mathcal{L}_{i, j, k, l}^{\omega}} .
\end{aligned}
$$

By Lemma 3.8, the volume of $\mathcal{H}\left(t_{i}, t_{l}, 1\right) \cap \mathcal{U}$ is bounded from below by $\frac{\pi}{12} x$. Since the volume of $\mathcal{U}$ is $\frac{4}{3} \pi R^{3}$, we get

$$
\operatorname{Pr}\left(p \notin \mathcal{H}\left(t_{i}, t_{l}, 1\right) \mid \mathbf{x}_{i, l}=x, \mathbf{y}_{i} \geqslant 1, \mathcal{L}_{i, j, k, l}^{\omega}\right)^{n-4}<\left(1-\frac{x}{16 R^{3}}\right)^{n-4} .
$$

For any $0 \leqslant t \leqslant 1$, we have $(1-t) \leqslant e^{-t}$ thus

$$
(1-t)^{n-4} \leqslant e^{-t(n-4)}=e^{-t n} e^{4 t} \leqslant e^{4} e^{-t n}<55 e^{-t n} .
$$

Now $0 \leqslant x \leqslant 2 R$ and $R \geqslant 1$ since $B_{i}$ is entirely inside $\mathcal{U}$. Thus $0 \leqslant \frac{x}{16 R^{3}} \leqslant \frac{1}{8 R^{2}} \leqslant 1$ and

$$
\begin{aligned}
\operatorname{Pr}\left(p \notin \mathcal{H}\left(t_{i}, t_{l}, 1\right) \mid \mathbf{x}_{i, l}=x, \mathbf{y}_{i} \geqslant 1, \mathcal{L}_{i, j, k, l}^{\omega}\right)^{n-4} & <55 \exp \left(-\frac{n x}{16 R^{3}}\right) \\
& =55 \exp \left(-\frac{\mu x}{16}\right)
\end{aligned}
$$

The following proposition now bounds the integral $I_{y \geqslant 1}$.

Proposition 3.10. $I_{y \geqslant 1}$ is $O\left(\frac{1}{n^{3}}\right)$.

Proof. Recall that

$$
I_{y \geqslant 1}=\int_{x=0}^{2 R} \operatorname{Pr}\left(\delta_{i, j, k, l}^{\omega} \mid \mathbf{x}_{i, l}=x, \mathbf{y}_{i} \geqslant 1\right) \cdot \operatorname{Pr}\left(x \leqslant \mathbf{x}_{i, l}<x+d x \mid \mathbf{y}_{i} \geqslant 1\right) .
$$

By Lemmas 3.5 and 3.9 we have

$$
\operatorname{Pr}\left(\delta_{i, j, k, l}^{\omega} \mid \mathbf{x}_{i, l}=x, \mathbf{y}_{i} \geqslant 1\right) \leqslant \frac{(3 x+8)^{2}}{R^{6}} \cdot 55 \exp \left(-\frac{\mu x}{16}\right) .
$$


Similarly as in Lemma 3.6 we have

$$
\operatorname{Pr}\left(x \leqslant \mathbf{x}_{i, l}<x+d x \mid \mathbf{y}_{i} \geqslant 1\right) \leqslant \frac{3 x^{2}}{R^{3}} d x
$$

Thus we get

$$
\begin{aligned}
I_{y \geqslant 1} & \leqslant \int_{x=0}^{2 R} \frac{(3 x+8)^{2}}{R^{6}} \cdot 55 \exp \left(-\frac{\mu x}{16}\right) \cdot \frac{3 x^{2}}{R^{3}} d x \\
& \leqslant \frac{\mu^{3}}{n^{3}} \int_{x=0}^{+\infty} 3 x^{2}(3 x+8)^{2} \cdot 55 \exp \left(-\frac{\mu x}{16}\right) d x .
\end{aligned}
$$

Changing $\frac{\mu x}{16}$ by $z$ we get integrals of the kind

$$
\int_{0}^{\infty} z^{r} \exp (-z) d z
$$

which is bounded by a constant and thus $I_{y \geqslant 1}$ is $O\left(\frac{1}{n^{3}}\right)$.

3.2.3. $B_{i}$ and $B_{l}$ are not close to one another and $B_{i}$ is partially outside $\mathcal{U}$. The only remaining task is to bound the integral $I_{x>6, y<1}$. As in the previous case, we need to bound from below the volume of $\mathcal{H}\left(t_{i}, t_{l}, 1\right) \cap \mathcal{U}$. Here, however, the tangent $t_{i} t_{l}$ can be entirely outside $\mathcal{U}$, so the bound of Lemma 3.8 does not apply and a more intricate proof is needed. We need to distinguish two cases depending on the distance of segment $t_{i} t_{l}$ from $O$, the center of $\mathcal{U}$.

To this aim, we introduce two new types of events. For any $s \in \mathbb{R}$, let $\mathcal{F}_{i, j, k, l}^{\omega}(s)$ (resp. $\left.\mathcal{N}_{i, j, k, l}^{\omega}(s)\right)$ be the event that $\mathcal{L}_{i, j, k, l}^{\omega}$ occurs and the line segment $t_{i} t_{l}$ is at distance greater (resp. less) than $R+1-s$ from $O$. For reasons that will become clear in the proof of Lemma 3.13, we consider $s=y^{\frac{2}{3}}$.

The next five lemmas are used to bound the first term of the integral $I_{x>6, y<1}$.

Lemma 3.11. For any random point $p$ in $\mathcal{U}, \operatorname{Pr}\left(\delta_{i, j, k, l}^{\omega} \mid \mathbf{x}_{i, l}=x, \mathbf{y}_{i}=y, \mathbf{y}_{l} \leqslant \mathbf{y}_{i}\right)$ is equal to

$$
\begin{aligned}
& \operatorname{Pr}\left(\mathcal{F}_{i, j, k, l}^{\omega}\left(y^{\frac{2}{3}}\right) \mid \mathbf{x}_{i, l}=x, \mathbf{y}_{i}=y, \mathbf{y}_{l} \leqslant \mathbf{y}_{i}\right) \\
& \cdot \operatorname{Pr}\left(p \notin \mathcal{H}\left(t_{i}, t_{l}, 1\right) \mid \mathbf{x}_{i, l}=x, \mathbf{y}_{i}=y, \mathbf{y}_{l} \leqslant \mathbf{y}_{i}, \mathcal{F}_{i, j, k, l}^{\omega}\left(y^{\frac{2}{3}}\right)\right)^{n-4} \\
& +\operatorname{Pr}\left(\mathcal{N}_{i, j, k, l}^{\omega}\left(y^{\frac{2}{3}}\right) \mid \mathbf{x}_{i, l}=x, \mathbf{y}_{i}=y, \mathbf{y}_{l} \leqslant \mathbf{y}_{i}\right) \\
& \cdot \operatorname{Pr}\left(p \notin \mathcal{H}\left(t_{i}, t_{l}, 1\right) \mid \mathbf{x}_{i, l}=x, \mathbf{y}_{i}=y, \mathbf{y}_{l} \leqslant \mathbf{y}_{i}, \mathcal{N}_{i, j, k, l}^{\omega}\left(y^{\frac{2}{3}}\right)\right)^{n-4} .
\end{aligned}
$$

Proof. $\delta_{i, j, k, l}^{\omega}$ implies $\mathcal{L}_{i, j, k, l}^{\omega}$ which can be split into $\mathcal{F}_{i, j, k, l}^{\omega}\left(y^{\frac{2}{3}}\right), \mathcal{N}_{i, j, k, l}^{\omega}\left(y^{\frac{2}{3}}\right)$, and the event that $\mathcal{L}_{i, j, k, l}^{\omega}$ occurs and the line segment $t_{i} t_{l}$ is at distance exactly $R+1-y^{\frac{2}{3}}$ from $O$. This later event occurs with probability 0 , thus

$$
\begin{aligned}
\operatorname{Pr}\left(\delta_{i, j, k, l}^{\omega} \mid \mathbf{x}_{i, l}=x, \mathbf{y}_{i}=y,\right. & \left.\mathbf{y}_{l} \leqslant \mathbf{y}_{i}\right)= \\
& \operatorname{Pr}\left(\delta_{i, j, k, l}^{\omega} \cap \mathcal{F}_{i, j, k, l}^{\omega}\left(y^{\frac{2}{3}}\right) \mid \mathbf{x}_{i, l}=x, \mathbf{y}_{i}=y, \mathbf{y}_{l} \leqslant \mathbf{y}_{i}\right) \\
& +\operatorname{Pr}\left(\delta_{i, j, k, l}^{\omega} \cap \mathcal{N}_{i, j, k, l}^{\omega}\left(y^{\frac{2}{3}}\right) \mid \mathbf{x}_{i, l}=x, \mathbf{y}_{i}=y, \mathbf{y}_{l} \leqslant \mathbf{y}_{i}\right),
\end{aligned}
$$


which can be expanded into

$$
\begin{aligned}
& \operatorname{Pr}\left(\mathcal{F}_{i, j, k, l}^{\omega}\left(y^{\frac{2}{3}}\right) \mid \mathbf{x}_{i, l}=x, \mathbf{y}_{i}=y, \mathbf{y}_{l} \leqslant \mathbf{y}_{i}\right) \\
& \cdot \operatorname{Pr}\left(\delta_{i, j, k, l}^{\omega} \mid \mathbf{x}_{i, l}=x, \mathbf{y}_{i}=y, \mathbf{y}_{l} \leqslant \mathbf{y}_{i}, \mathcal{F}_{i, j, k, l}^{\omega}\left(y^{\frac{2}{3}}\right)\right) \\
& +\operatorname{Pr}\left(\mathcal{N}_{i, j, k, l}^{\omega}\left(y^{\frac{2}{3}}\right) \mid \mathbf{x}_{i, l}=x, \mathbf{y}_{i}=y, \mathbf{y}_{l} \leqslant \mathbf{y}_{i}\right) \\
& \cdot \operatorname{Pr}\left(\delta_{i, j, k, l}^{\omega} \mid \mathbf{x}_{i, l}=x, \mathbf{y}_{i}=y, \mathbf{y}_{l} \leqslant \mathbf{y}_{i}, \mathcal{N}_{i, j, k, l}^{\omega}\left(y^{\frac{2}{3}}\right)\right) .
\end{aligned}
$$

When $\mathcal{F}_{i, j, k, l}^{\omega}\left(y^{\frac{2}{3}}\right)$ occurs, the probability

$$
\operatorname{Pr}\left(\delta_{i, j, k, l}^{\omega} \mid \mathbf{x}_{i, l}=x, \mathbf{y}_{i}=y, \mathbf{y}_{l} \leqslant \mathbf{y}_{i}, \mathcal{F}_{i, j, k, l}^{\omega}\left(y^{\frac{2}{3}}\right)\right)
$$

is the probability that the tangent is not occluded, that is, $p_{\gamma}$ does not belong to $\mathcal{H}\left(t_{i}, t_{l}, 1\right)$ for all the $n-4$ values of $\gamma \neq i, j, k, l$. The same argument holds for $\mathcal{N}_{i, j, k, l}^{\omega}\left(y^{\frac{2}{3}}\right)$. Since the $p_{\gamma}$ are independent, we get the result.

In order to bound the two terms in Lemma 3.11,

$$
\begin{aligned}
& \operatorname{Pr}\left(p \notin \mathcal{H}\left(t_{i}, t_{l}, 1\right) \mid \mathbf{x}_{i, l}=x, \mathbf{y}_{i}=y, \mathbf{y}_{l} \leqslant \mathbf{y}_{i}, \mathcal{F}_{i, j, k, l}^{\omega}\left(y^{\frac{2}{3}}\right)\right)^{n-4} \text { and } \\
& \operatorname{Pr}\left(p \notin \mathcal{H}\left(t_{i}, t_{l}, 1\right) \mid \mathbf{x}_{i, l}=x, \mathbf{y}_{i}=y, \mathbf{y}_{l} \leqslant \mathbf{y}_{i}, \mathcal{N}_{i, j, k, l}^{\omega}\left(y^{\frac{2}{3}}\right)\right)^{n-4},
\end{aligned}
$$

we need to bound the volume of $\mathcal{H}\left(t_{i}, t_{l}, 1\right) \cap \mathcal{U}$ from below.

LEMma 3.12. When $\mathbf{x}_{i, l} \geqslant 6, \mathbf{y}_{l} \leqslant \mathbf{y}_{i} \leqslant 1, \mathcal{L}_{i, j, k, l}^{\omega}$ occurs and segment $t_{i} t_{l}$ is at distance less than $R+1-s, 0 \leqslant s \leqslant 1$, from the center of $\mathcal{U}$, then the volume of $\mathcal{H}\left(t_{i}, t_{l}, 1\right) \cap \mathcal{U}$ is larger than $\frac{1}{6 \sqrt{2}}\left(\mathbf{x}_{i, l}-5\right) s \sqrt{s}$.

Proof. We give here the idea of the proof; full details can be found in Appendix A. Let $t$ be the closest point on segment $t_{i} t_{l}$ from $O$, and $D$ be a unit radius disk centered at $t$ in a plane containing $O$, the center of $\mathcal{U}$. We define a quadrilateral with vertices $a, b, a^{\prime}, b^{\prime}$ such that $a$ and $a^{\prime}$ are the closest and the farthest points, respectively, in $D \cap \mathcal{U}$ from $O$, and $b$ and $b^{\prime}$ are the points of intersection of $\partial D$ and the perpendicular bisector of segment $a a^{\prime}$ (see Figure 3.2). Let $v$ be equal to $R+1$ minus the distance from $O$ to segment $t_{i} t_{l}$. We prove that the convex hull of $a, b, a^{\prime}, b^{\prime}$ and $p_{l}$, which is included in $\mathcal{H}\left(t_{i}, t_{l}, 1\right) \cap \mathcal{U}$, has volume greater than $\frac{1}{6 \sqrt{2}}\left(\mathbf{x}_{i, l}-5\right) \min (2 \sqrt{2}, v \sqrt{v})$. It follows that, for any $0 \leqslant s \leqslant 1$, if segment $t_{i} t_{l}$ is at distance less than $R+1-s$ from $O$, then $v \geqslant s$ and the volume of $\mathcal{H}\left(t_{i}, t_{l}, 1\right) \cap \mathcal{U}$ is greater than $\frac{1}{6 \sqrt{2}}\left(\mathbf{x}_{i, l}-5\right) s \sqrt{s}$. $\square$

LEMмA 3.13. For any random point $p$ in $\mathcal{U}, x \geqslant 6$ and $0 \leqslant y \leqslant 1$,

$$
\begin{aligned}
\operatorname{Pr}\left(p \notin \mathcal{H}\left(t_{i}, t_{l}, 1\right) \mid \mathbf{x}_{i, l}=x, \mathbf{y}_{i}=y, \mathbf{y}_{l} \leqslant \mathbf{y}_{i}, \mathcal{F}_{i, j, k, l}^{\omega}\left(y^{\frac{2}{3}}\right)\right)^{n-4} & \\
& <55 \exp \left(-\frac{\mu(x-5) y^{2}}{8 \sqrt{2} \pi}\right)
\end{aligned}
$$

and

$$
\begin{aligned}
\operatorname{Pr}\left(p \notin \mathcal{H}\left(t_{i}, t_{l}, 1\right) \mid \mathbf{x}_{i, l}=x, \mathbf{y}_{i}=y, \mathbf{y}_{l} \leqslant \mathbf{y}_{i}, \mathcal{N}_{i, j, k, l}^{\omega}\left(y^{\frac{2}{3}}\right)\right)^{n-4} & \\
& <55 \exp \left(-\frac{\mu(x-5) y}{8 \sqrt{2} \pi}\right) .
\end{aligned}
$$




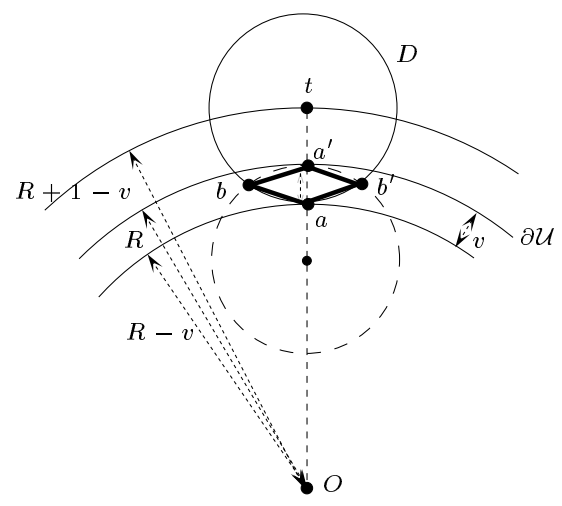

Fig. 3.2. For the sketch of the proof of Lemma $3.12(v \in(0,1))$.

Furthermore, if $x \geqslant 6 \sqrt{R}$ then

$$
\begin{aligned}
\operatorname{Pr}\left(p \notin \mathcal{H}\left(t_{i}, t_{l}, 1\right) \mid \mathbf{x}_{i, l}=x, \mathbf{y}_{i}=y, \mathbf{y}_{l} \leqslant \mathbf{y}_{i}, \mathcal{N}_{i, j, k, l}^{\omega}\left(y^{\frac{2}{3}}\right)\right)^{n-4} & \\
& <55 \exp \left(-\frac{\mu(x-5)}{8 \sqrt{2} \pi}\right) .
\end{aligned}
$$

Proof. Let $\mathbf{x}_{i, l}=x, \mathbf{y}_{i}=y$ and suppose first that event $\mathcal{F}_{i, j, k, l}^{\omega}\left(y^{\frac{2}{3}}\right)$ occurs. Since $p_{i}$ is at distance $R-y$ from $O$, the segment $t_{i} t_{l}$ is at distance less than $R+1-y$ from $O$, and thus, by Lemma 3.12 , the volume of $\mathcal{H}\left(t_{i}, t_{l}, 1\right) \cap \mathcal{U}$ is greater than $\frac{1}{6 \sqrt{2}}(x-5) y \sqrt{y}$, which is bigger than $\frac{1}{6 \sqrt{2}}(x-5) y^{2}$ since $0 \leqslant y \leqslant 1$ (we bound $y \sqrt{y}$ from below by $y^{2}$ only so that we can actually compute the integral $\mathcal{I}_{1}$ in the proof of Proposition 3.18). We now follow the proof of Lemma 3.9, except that the volume of $\mathcal{H}\left(t_{i}, t_{l}, 1\right) \cap \mathcal{U}$ is now bounded from below by $\frac{1}{6 \sqrt{2}}(x-5) y^{2}$ instead of $\frac{\pi}{12} x$. We get

$$
\begin{aligned}
\operatorname{Pr}\left(p \notin \mathcal{H}\left(t_{i}, t_{l}, 1\right) \mid \mathbf{x}_{i, l}=x, \mathbf{y}_{i}=y, \mathbf{y}_{l} \leqslant \mathbf{y}_{i}, \mathcal{F}_{i, j, k, l}^{\omega}\left(y^{\frac{2}{3}}\right)\right)^{n-4} & \\
& <55 \exp \left(-\frac{\mu(x-5) y^{2}}{8 \sqrt{2} \pi}\right) .
\end{aligned}
$$

When $\mathcal{N}_{i, j, k, l}^{\omega}\left(y^{\frac{2}{3}}\right)$ occurs, the segment $t_{i} t_{l}$ is at distance less than $R+1-y^{\frac{2}{3}}$ from $O$, and thus, by Lemma 3.12, the volume of $\mathcal{H}\left(t_{i}, t_{l}, 1\right) \cap \mathcal{U}$ is bounded from below by $\frac{1}{6 \sqrt{2}}(x-5) y^{\frac{2}{3}} \sqrt{y^{\frac{2}{3}}}=\frac{1}{6 \sqrt{2}}(x-5) y$. Then, as before, we get

$$
\begin{aligned}
\operatorname{Pr}\left(p \notin \mathcal{H}\left(t_{i}, t_{l}, 1\right) \mid \mathbf{x}_{i, l}=x, \mathbf{y}_{i}=y, \mathbf{y}_{l} \leqslant \mathbf{y}_{i}, \mathcal{N}_{i, j, k, l}^{\omega}\left(y^{\frac{2}{3}}\right)\right)^{n-4} & \\
& <55 \exp \left(-\frac{\mu(x-5) y}{8 \sqrt{2} \pi}\right) .
\end{aligned}
$$

Now, if $x \geqslant 6 \sqrt{R}$, the length of the tangent $t_{i} t_{l}$ is at least $6 \sqrt{R}-2$. Since $x \geqslant 6, R>3$ and a simple computation shows that $6 \sqrt{R}-2$ is bigger than $2 \sqrt{2 R+1}$ which is the length of the longest line segment that may entirely lie inside $\mathcal{U}^{+} \backslash \mathcal{U}$. Thus $\operatorname{dist}\left(O, t_{i} t_{l}\right) \leqslant R=R+1-s$ with $s=1$ and, by Lemma 3.12 , the volume of 
$\mathcal{H}\left(t_{i}, t_{l}, 1\right) \cap \mathcal{U}$ is greater than $\frac{1}{6 \sqrt{2}}(x-5)$. Then, as before, we get

$$
\begin{aligned}
\operatorname{Pr}\left(p \notin \mathcal{H}\left(t_{i}, t_{l}, 1\right) \mid \mathbf{x}_{i, l}=x, \mathbf{y}_{i}=y, \mathbf{y}_{l} \leqslant \mathbf{y}_{i}, \mathcal{N}_{i, j, k, l}^{\omega}\left(y^{\frac{2}{3}}\right)\right)^{n-4} & \\
& <55 \exp \left(-\frac{\mu(x-5)}{8 \sqrt{2} \pi}\right) .
\end{aligned}
$$

Lemma 3.14. $\operatorname{Pr}\left(\mathcal{N}_{i, j, k, l}^{\omega}\left(y^{\frac{2}{3}}\right) \mid \mathbf{x}_{i, l}=x, \mathbf{y}_{i}=y, \mathbf{y}_{l} \leqslant \mathbf{y}_{i}\right) \leqslant \frac{(3 x+8)^{2}}{R^{6}}$.

Proof. The event $\mathcal{N}_{i, j, k, l}^{\omega}\left(y^{\frac{2}{3}}\right)$ occurs only if $\mathcal{L}_{i, j, k, l}^{\omega}$ occurs. The result thus follows since, by Lemmas 3.3 and 3.4, $\operatorname{Pr}\left(\mathcal{L}_{i, j, k, l}^{\omega} \mid \mathbf{x}_{i, l}=x, \mathbf{y}_{i}=y, \mathbf{y}_{l} \leqslant \mathbf{y}_{i}\right) \leqslant \frac{(3 x+8)^{2}}{R^{6}}$. $\mathbf{u}$

Lemma 3.15. If $y<1$, then

$$
\operatorname{Pr}\left(\mathcal{F}_{i, j, k, l}^{\omega}\left(y^{\frac{2}{3}}\right) \mid \mathbf{x}_{i, l}=x, \mathbf{y}_{i}=y\right) \leqslant 81 \pi^{2} \frac{(x+6)^{2} y^{2}}{R^{6}} .
$$

Proof. A "far" tangent $t_{i} t_{l}$ is at distance at least $R+1-y^{\frac{2}{3}}$ from the center $O$ of $\mathcal{U}$. Such a segment also lies in $\mathcal{H}\left(p_{i}, p_{l}, 1\right)$. Let $E$ be the part of $\mathcal{H}\left(p_{i}, p_{l}, 1\right)$ lying outside of the sphere of radius $R+1-y^{\frac{2}{3}}$ and center $O$. See Figure 3.3 (a). Now, both $p_{j}$ and $p_{k}$ must be in the region inside $\mathcal{U}$ and within distance 1 from $E$. Denote this region by $K$. Then

$$
\operatorname{Pr}\left(\mathcal{F}_{i, j, k, l}^{\omega}\left(y^{\frac{2}{3}}\right) \mid \mathbf{x}_{i, l}=x, \mathbf{y}_{i}=y, \mathbf{y}_{l} \leqslant \mathbf{y}_{i}\right) \leqslant\left(\frac{\text { Volume of } K}{\text { Volume of } \mathcal{U}}\right)^{2} .
$$

By Proposition B.1, which we prove in Appendix B, the volume of $K$ is bounded from above by $12 \pi^{2}(x+6) y$, which yields the result. Here we give the intuition of the proof. Refer to Figure 3.3. First notice that the "length" of $K$ is at most $x+4$. Since $K$ is enclosed in between a sphere of radius $R$ and one of radius $R-y^{\frac{2}{3}}$, its "height" is at most $y^{\frac{2}{3}}$. For the "width", consider Figure 3.3 (b) which shows a cross-section of $K$ taken with a plane through $O$ and perpendicular to $p_{i} p_{l}$. The "width" of $K$ is no more than 2 times the "width" of $E$. The "height" of $E$ can be bounded by some constant times $y^{\frac{2}{3}}$; thus its "width" can be bounded by some constant times $\sqrt{y^{\frac{2}{3}}}=y^{\frac{1}{3}}$. Thus, intuitively, the volume of $K$ is smaller than $(x+4) y^{\frac{2}{3}} y^{\frac{1}{3}}=(x+4) y$, up to a constant, and the result follows.

We now bound the two last terms of the integral $I_{x>6, y<1}$.

Lemma 3.16. $\operatorname{Pr}\left(y \leqslant \mathbf{y}_{i}<y+d y\right) \leqslant \frac{3 d y}{R}$.

Proof. The event $\left(y \leqslant \mathbf{y}_{i}<y+d y\right)$ occurs only if $p_{i}$ lies in the spherical shell delimited by the two spheres centered at $O$ of radii $R-y$ and $R-y-d y$ whose volume is smaller than $4 \pi R^{2} d y$. Dividing by the volume of $\mathcal{U}$ proves the result.

Lemma 3.17. For $6 \leqslant x \leqslant 2 R$ and $y \leqslant 1$, we have

$$
\operatorname{Pr}\left(\left(x \leqslant \mathbf{x}_{i, l}<x+d x\right) \cap\left(\mathbf{y}_{l} \leqslant \mathbf{y}_{i}\right) \mid \mathbf{y}_{i}=y\right) \leqslant \frac{6 x y d x}{R^{3}} .
$$

Proof. The probability $\operatorname{Pr}\left(\left(x \leqslant \mathbf{x}_{i, l}<x+d x\right) \cap\left(\mathbf{y}_{l} \leqslant \mathbf{y}_{i}\right) \mid \mathbf{y}_{i}=y\right)$ is equal to the volume of the region (shown in grey in Figure 3.4) which is the intersection of the region in between the two spheres centered at $p_{i}$ and of radii $x$ and $x+d x$, and the 


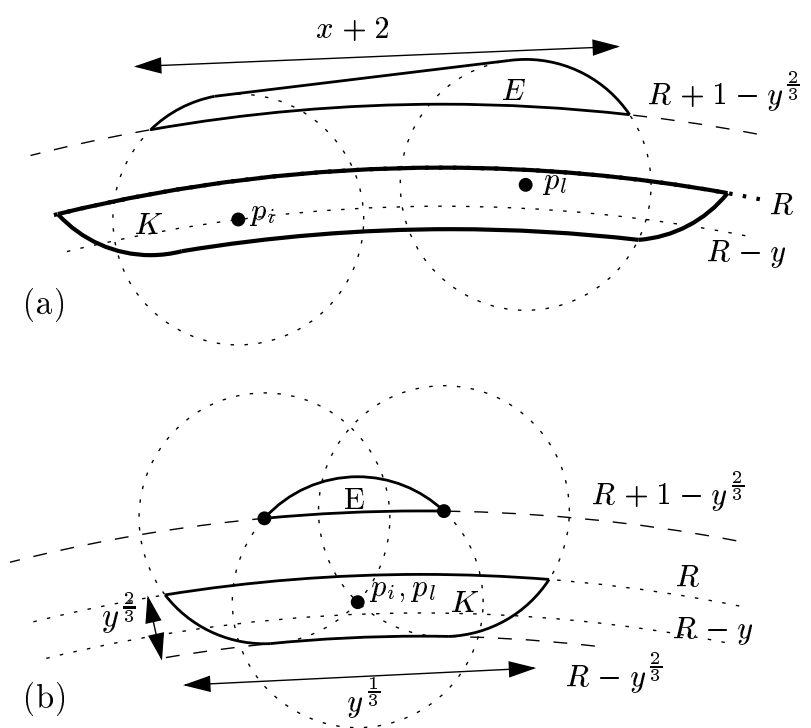

Fig. 3.3. For the sketch of the proof of Lemma 3.15 .

region in between the two spheres centered at $O$ and of radii $R$ and $R-y$, divided by the volume of $\mathcal{U}$. We prove in Proposition C.1 in Appendix $\mathrm{C}$ that the volume of that region is at most $8 \pi x y d x$. Roughly speaking, the volume bounded by the four spheres is at most $8 \pi x y d x$ because, its "thickness" is $d x$, its "height" is $y$ and its "radius" is $x$. Dividing by the volume of $\mathcal{U}$ proves the result. $\square$

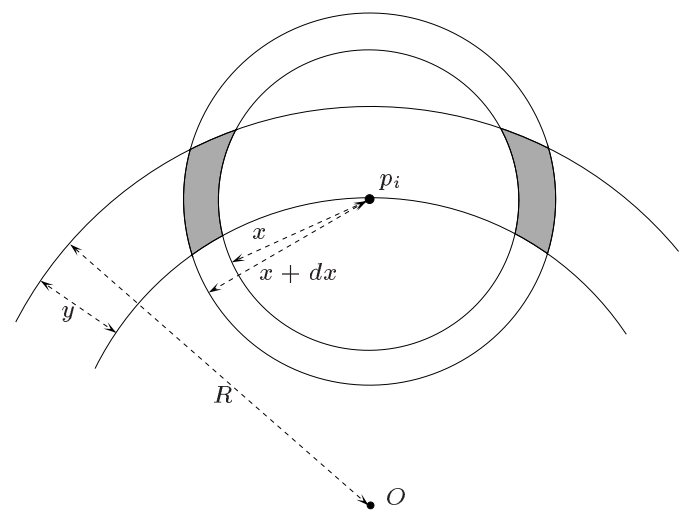

Fig. 3.4. For the proof of Lemma 3.17 .

We can now bound the integral $I_{x>6, y<1}$ of Lemma 3.2.

Proposition 3.18. $I_{x>6, y<1}$ is $O\left(\frac{1}{n^{3}}\right)$.

Proof. Recall that

$$
\begin{aligned}
I_{x>6, y<1}=\int_{x=6}^{2 R} \int_{y=0}^{1} & \operatorname{Pr}\left(\delta_{i, j, k, l}^{\omega} \mid \mathbf{x}_{i, l}=x, \mathbf{y}_{i}=y, \mathbf{y}_{l} \leqslant \mathbf{y}_{i}\right) \\
& \operatorname{Pr}\left(\left(x \leqslant \mathbf{x}_{i, l}<x+d x\right) \cap\left(\mathbf{y}_{l} \leqslant \mathbf{y}_{i}\right) \mid \mathbf{y}_{i}=y\right) \\
& \operatorname{Pr}\left(y \leqslant \mathbf{y}_{i}<y+d y\right) .
\end{aligned}
$$


By Lemmas 3.16 and 3.17 , we get

$$
I_{x>6, y<1} \leqslant \int_{x=6}^{2 R} \int_{y=0}^{1} \operatorname{Pr}\left(\delta_{i, j, k, l}^{\omega} \mid \mathbf{x}_{i, l}=x, \mathbf{y}_{i}=y, \mathbf{y}_{l} \leqslant \mathbf{y}_{i}\right) \cdot \frac{6 x y d x}{R^{3}} \cdot \frac{3 d y}{R} .
$$

By Lemma $3.11, \operatorname{Pr}\left(\delta_{i, j, k, l}^{\omega} \mid \mathbf{x}_{i, l}=x, \mathbf{y}_{i}=y, \mathbf{y}_{l} \leqslant \mathbf{y}_{i}\right)$ is equal to

$$
\begin{aligned}
& \operatorname{Pr}\left(\mathcal{F}_{i, j, k, l}^{\omega}\left(y^{\frac{2}{3}}\right) \mid \mathbf{x}_{i, l}=x, \mathbf{y}_{i}=y, \mathbf{y}_{l} \leqslant \mathbf{y}_{i}\right) \\
& \cdot \operatorname{Pr}\left(p \notin \mathcal{H}\left(t_{i}, t_{l}, 1\right) \mid \mathbf{x}_{i, l}=x, \mathbf{y}_{i}=y, \mathbf{y}_{l} \leqslant \mathbf{y}_{i}, \mathcal{F}_{i, j, k, l}^{\omega}\left(y^{\frac{2}{3}}\right)\right)^{n-4} \\
& +\operatorname{Pr}\left(\mathcal{N}_{i, j, k, l}^{\omega}\left(y^{\frac{2}{3}}\right) \mid \mathbf{x}_{i, l}=x, \mathbf{y}_{i}=y, \mathbf{y}_{l} \leqslant \mathbf{y}_{i}\right) \\
& \cdot \operatorname{Pr}\left(p \notin \mathcal{H}\left(t_{i}, t_{l}, 1\right) \mid \mathbf{x}_{i, l}=x, \mathbf{y}_{i}=y, \mathbf{y}_{l} \leqslant \mathbf{y}_{i}, \mathcal{N}_{i, j, k, l}^{\omega}\left(y^{\frac{2}{3}}\right)\right)^{n-4} .
\end{aligned}
$$

We split the integral at $x=6 \sqrt{R}$. When $x \geqslant 6 \sqrt{R}$, the distance from $O$ to the tangent $t_{i} t_{l}$ is less than $R$ (see the proof of Lemma 3.13), which is less than $R+1-y^{\frac{2}{3}}$ for any $y$ in $(0,1)$. Thus, for any $x \geqslant 6 \sqrt{R}$ and $y \in(0,1)$, the probability $\operatorname{Pr}\left(\mathcal{F}_{i, j, k, l}^{\omega}\left(y^{\frac{2}{3}}\right) \mid \mathbf{x}_{i, l}=x, \mathbf{y}_{i}=y, \mathbf{y}_{l} \leqslant \mathbf{y}_{i}\right)$ is equal to 0 . It then follows from Lemmas $3.13,3.14$ and 3.15 that $I_{x>6, y<1} \leqslant \mathcal{I}_{1}+\mathcal{I}_{2}+\mathcal{I}_{3}$ with

$$
\begin{aligned}
& \mathcal{I}_{1}=\int_{x=6}^{6 \sqrt{R}} \int_{y=0}^{1} 81 \pi^{2} \frac{(x+6)^{2} y^{2}}{R^{6}} \cdot 55 \exp \left(-\frac{\mu(x-5) y^{2}}{8 \sqrt{2} \pi}\right) \cdot \frac{6 x y d x}{R^{3}} \cdot \frac{3 d y}{R}, \\
& \mathcal{I}_{2}=\int_{x=6}^{6 \sqrt{R}} \int_{y=0}^{1} \frac{(3 x+8)^{2}}{R^{6}} \cdot 55 \exp \left(-\frac{\mu(x-5) y}{8 \sqrt{2} \pi}\right) \cdot \frac{6 x y d x}{R^{3}} \cdot \frac{3 d y}{R}, \\
& \mathcal{I}_{3}=\int_{x=6 \sqrt{R}}^{2 R} \int_{y=0}^{1} \frac{(3 x+8)^{2}}{R^{6}} \cdot 55 \exp \left(-\frac{\mu(x-5)}{8 \sqrt{2} \pi}\right) \cdot \frac{6 x y d x}{R^{3}} \cdot \frac{3 d y}{R} .
\end{aligned}
$$

Changing $\frac{\mu(x-5)}{8 \sqrt{2} \pi}$ by $z$ in the three integrals and $y^{2}$ by $y^{\prime}$ in $\mathcal{I}_{1}$, we get

$$
\begin{aligned}
& \mathcal{I}_{1} \leqslant \frac{K}{R^{10}} \sum_{u=0}^{u=3} \int_{z=0}^{c \sqrt{R}} \int_{y^{\prime}=0}^{1} z^{u} y^{\prime} \exp \left(-z y^{\prime}\right) d z d y^{\prime} \\
& \mathcal{I}_{2} \leqslant \frac{K}{R^{10}} \sum_{u=0}^{u=3} \int_{z=0}^{c \sqrt{R}} \int_{y=0}^{1} z^{u} y \exp (-z y) d z d y \\
& \mathcal{I}_{3} \leqslant \frac{K}{R^{10}} \sum_{u=0}^{u=3} \int_{z=0}^{\infty} \int_{y=0}^{1} z^{u} y \exp (-z) d z d y
\end{aligned}
$$

where $K$ and $c$ are some positive constants.

Note first that $\mathcal{I}_{3}$ is bounded from above by $\frac{K}{R^{10}} \sum_{u=0}^{u=3} \int_{z=0}^{\infty} z^{u} \exp (-z) d z$. These integrals are bounded by a constant, thus $\mathcal{I}_{3}$ is $O\left(\frac{1}{R^{10}}\right)$.

To bound the integrals $\mathcal{I}_{1}$ and $\mathcal{I}_{2}$, we now compute the integral

$$
\int_{z=0}^{A} \int_{y=0}^{1} z^{u} y \exp (-z y) d z d y
$$

for $u \in\{0, \ldots, 3\}$ and $A>0$, for example with Maple [16]. For $u=0$ it is equal to

$$
\frac{\exp (-A)+A-1}{A} \text {. }
$$


For $u=1$, the integral (3.2) is equal to

$$
\exp (-A)+\ln A+E i(1, A)+\gamma-1
$$

where $\operatorname{Ei}(1, A)$ denotes the exponential integral $\int_{t=1}^{\infty} \frac{\exp (-A t)}{t} d t$ and $\gamma$ denotes Euler's constant. Finally, for $u=2$ or 3 , the integral (3.2) is equal to

$$
\exp (-A) P_{1}(A, u-1)+P_{2}(A, u-1)
$$

where $P_{i}(A, u-1)$ denotes a polynomial of degree $u-1$ in $A$.

When $A$ tends to $\infty,(3.3)$ tends to $1,(3.4)$ is equivalent to $\ln A$ (since $\operatorname{Ei}(1, A)$ tends to 0$)$ and (3.5) is equivalent to the leading monomial of $P_{2}(A, u-1)$ which is of degree $u-1 \leqslant 2$. This guarantees that for $A=c \sqrt{R}$ and $u \in\{0, \ldots, 3\}$, the integral (3.2) is $O(R)$. It follows that $\mathcal{I}_{1}$ and $\mathcal{I}_{2}$ are $O\left(\frac{1}{R^{9}}\right)$.

Since $R^{3}=n / \mu$, we get that $I_{x>6, y<1} \leqslant \mathcal{I}_{1}+\mathcal{I}_{2}+\mathcal{I}_{3}=O\left(\frac{1}{R^{9}}\right)=O\left(\frac{1}{n^{3}}\right)$.

We can now conclude the proof that the expected number of $T 4$-segments is $O(n)$, because, by Lemmas 3.1, 3.2, and Propositions 3.7, 3.10, and 3.18, the expected number of $T 4$-segments is smaller than

$$
\sum_{(i, j, k, l) \in \mathcal{N}} \sum_{\omega=1}^{12}\left(O\left(\frac{1}{n^{3}}\right)+O\left(\frac{1}{n^{3}}\right)+O\left(\frac{1}{n^{3}}\right)\right)=O(n) .
$$

4. The expected number of $T 4$-segments is at least linear. In this section, we prove that the expected number of $T 4$-segments amongst $n$ uniformly distributed unit balls is $\Omega(n)$. To do this, we bound from below the probability that four given balls have a given $T 4$-segment. The key step is to give a condition on the relative positions of four unit balls that guarantees that they have exactly twelve common tangent lines. We use here the notation as defined in Section 3.1.

Lemma 4.1. Let $e$ be a real number satisfying $\frac{4 \sqrt{2}}{3}<e<2$ and let the radius $R$ of $\mathcal{U}$ be strictly greater than $e$. There exists an $\epsilon>0$ such that for any point $p \in \mathcal{U}$, there exist three balls $\Gamma_{1}(p), \Gamma_{2}(p), \Gamma_{3}(p)$ of radius $\epsilon$ contained in $\mathcal{U}$ and satisfying the following conditions:

- $p$ and the centers of the $\Gamma_{i}(p)$ form a regular tetrahedron with edges of length e, and

- for any triple of points $\left(p_{1}, p_{2}, p_{3}\right), p_{i}$ taken from $\Gamma_{i}(p)$, the four unit balls centered at $p, p_{1}, p_{2}$ and $p_{3}$ have exactly 12 distinct tangent lines.

Proof. Macdonald, Pach and Theobald proved [15, Lemma 3] that 4 unit balls centered on the vertices of a regular tetrahedron with edges of length $e, \frac{4 \sqrt{2}}{3}<e<2$, have exactly 12 distinct real common tangent lines. Moreover, these 12 tangent lines correspond to the 12 real roots of a system of equations of degree 12, thus each tangent line corresponds to a simple root of that system of equations. It thus follows that for any sufficiently small perturbation of the 4 ball centers, the 4 perturbed balls still have 12 real common tangent lines. Let $\epsilon>0$ be such that the 4 ball centers can move distance $\epsilon$ in any direction while keeping 12 distinct common tangents.

Now, for any point $p \in \mathcal{U}$, consider a regular tetrahedron with edge length $e$ having $p$ as a vertex and such that the other vertices are at distance at least $\epsilon$ from the boundary of $\mathcal{U}$; for example, we can choose the other three vertices on a plane perpendicular to the segment $O p$. Let $\Gamma_{1}(p), \Gamma_{2}(p)$, and $\Gamma_{3}(p)$ be the balls of radius $\epsilon$ centered at the vertices, distinct from $p$, of that tetrahedron. By the previous reasoning, for any $q \in \Gamma_{1}(p), r \in \Gamma_{2}(p)$, and $s \in \Gamma_{3}(p)$, the four unit balls centered at $p, q, r$ and $s$ have exactly twelve tangents. 
Now, by Lemma 3.1, the expected number of $T 4$-segments is

$$
\sum_{(i, j, k, l) \in \mathcal{N}} \sum_{\omega=1}^{12} \operatorname{Pr}\left(\delta_{i, j, k, l}^{\omega}\right)
$$

Thus we only need to bound from below the probability that the event $\delta_{i, j, k, l}^{\omega}$ occurs.

Lemma 4.2. $\operatorname{Pr}\left(\delta_{i, j, k, l}^{\omega}\right)$ is $\Omega\left(\frac{1}{n^{3}}\right)$.

Proof. Assume that $n>8 \mu$ so that the radius $R=\sqrt[3]{n / \mu}$ of $\mathcal{U}$ is larger than 2 and let $T(p)$ be the set $\Gamma_{1}(p) \times \Gamma_{2}(p) \times \Gamma_{3}(p)$ where $\Gamma_{i}(p)$ and $e$ are defined as in Lemma 4.1. First, note that

$$
\begin{aligned}
\operatorname{Pr}\left(\delta_{i, j, k, l}^{\omega}\right) & \geqslant \operatorname{Pr}\left(\delta_{i, j, k, l}^{\omega} \cap\left(p_{i}, p_{j}, p_{k}\right) \in T\left(p_{l}\right)\right) \\
& =\operatorname{Pr}\left(\left(p_{i}, p_{j}, p_{k}\right) \in T\left(p_{l}\right)\right) \cdot \operatorname{Pr}\left(\delta_{i, j, k, l}^{\omega} \mid\left(p_{i}, p_{j}, p_{k}\right) \in T\left(p_{l}\right)\right) .
\end{aligned}
$$

Since $\Gamma_{1}\left(p_{l}\right), \Gamma_{2}\left(p_{l}\right)$, and $\Gamma_{3}\left(p_{l}\right)$ are three balls of radius $\epsilon$ entirely contained in $\mathcal{U}$, we have

$$
\operatorname{Pr}\left(\left(p_{i}, p_{j}, p_{k}\right) \in T\left(p_{l}\right)\right)=\left(\frac{\frac{4}{3} \pi \epsilon^{3}}{\frac{4}{3} \pi R^{3}}\right)^{3}=\frac{\mu^{3} \epsilon^{9}}{n^{3}} .
$$

By Lemmas 3.3 and 4.1 , the event $\left(\delta_{i, j, k, l}^{\omega} \mid\left(p_{i}, p_{j}, p_{k}\right) \in T\left(p_{l}\right)\right)$ occurs if and only if the interior of $\mathcal{H}\left(t_{i}, t_{l}, 1\right) \cap \mathcal{U}$ does not contain the center of any ball. Note that the volume of $\mathcal{H}\left(t_{i}, t_{l}, 1\right) \cap \mathcal{U}$ is at most the volume of $\mathcal{H}\left(t_{i}, t_{l}, 1\right)$, which is at most $\frac{4}{3} \pi+\pi(2+e+2 \epsilon)$ since the length of $t_{i} t_{l}$ is at most $e+2+2 \epsilon$. It follows that

$$
\operatorname{Pr}\left(\delta_{i, j, k, l}^{\omega} \mid\left(p_{i}, p_{j}, p_{k}\right) \in T\left(p_{l}\right)\right) \geqslant\left(1-\frac{\pi\left(\frac{4}{3}+2+e+2 \epsilon\right)}{\operatorname{Volume}(\mathcal{U})}\right)^{n-4} .
$$

Since $e<2$, we get, after some elementary calculations, that

$$
\operatorname{Pr}\left(\delta_{i, j, k, l}^{\omega} \mid\left(p_{i}, p_{j}, p_{k}\right) \in T\left(p_{l}\right)\right) \geqslant\left(1-\frac{(6+2 \epsilon) \mu}{n}\right)^{n-4} .
$$

We thus have

$$
\operatorname{Pr}\left(\delta_{i, j, k, l}^{\omega}\right) \geqslant \frac{\mu^{3} \epsilon^{9}}{n^{3}}\left(1-\frac{(6+2 \epsilon) \mu}{n}\right)^{n-4} .
$$

Since $\left(1-\frac{(6+2 \epsilon) \mu}{n}\right)^{n-4}$ tends to $e^{-(6+2 \epsilon) \mu}$ when $n$ tends to infinity, we get

$$
\operatorname{Pr}\left(\delta_{i, j, k, l}^{\omega}\right)=\Omega\left(\frac{1}{n^{3}}\right)
$$

This completes the proof of the lower bound of Theorem 2.1 since the expected number of $T 4$-segments amongst $n$ uniformly distributed unit balls is, by Lemmas 3.1 and 4.2 ,

$$
\sum_{(i, j, k, l) \in \mathcal{N}} \sum_{\omega=1}^{12} \operatorname{Pr}\left(\delta_{i, j, k, l}^{\omega}\right)=\sum_{(i, j, k, l) \in \mathcal{N}} \sum_{\omega=1}^{12} \Omega\left(\frac{1}{n^{3}}\right)=\Omega(n) .
$$


5. The expected size of the visibility complex is linear. In this section we prove Theorem 2.2, that the expected size of the visibility complex of a set of $n$ uniformly distributed unit balls is linear.

We say that the balls are in general position if any $k$-dimensional face of the visibility complex is a connected set of maximal free segments tangent to exactly $4-k$ balls. We can assume that the balls are in general position since this occurs with probability 1 . We give a bound on the expected number of $k$-faces, for $k=0, \ldots, 4$.

Lemma 5.1. The expected number of 0 -faces is $\Theta(n)$.

Proof. A 0 -face of the visibility complex is a maximal free line segment tangent to 4 balls. Each maximal free line segment tangent to 4 balls contains a $T 4$-segment and each $T 4$-segment is contained in one maximal free line segment. Thus, by Theorem 2.1, the expected number of 0 -faces is linear. $\square$

To deal with the faces of dimension $k \geqslant 1$, we divide them into two classes. A $k$ face is open if it is incident to at least one $(k-1)$-face, otherwise it is closed. When the balls are in general position, the number of $k$-faces incident to a particular $(k-1)$-face is constant. In the proof of the following lemmas, any constant can be used. However, for completeness, we will use the exact values, but without justifying them.

Lemma 5.2. The expected number of 1-faces is $\Theta(n)$.

Proof. Note that a 0 -face corresponds to a maximal free segment tangent to 4 balls and it is incident to those 1-faces corresponding to free segments tangent to 3 amongst those 4 balls. So, a 0 -face is incident to exactly six 1 -faces, which implies that the number of open 1-faces is 6 times the number of 0 -faces, and is thus $\Theta(n)$ by the previous lemma.

Proving that the expected number of closed 1-faces is $O(n)$ can be done in a way very similar to the proof of the upper bound in Theorem 2.1. The difference is that we consider now only three balls and thus in all proofs, we forget ball $B_{k}$. We have to consider only $\left(\begin{array}{c}n \\ 3\end{array}\right)$ triples of balls instead of $\left(\begin{array}{c}n \\ 4\end{array}\right)$ quadruples, but we remove from the integral the probability $\operatorname{Pr}\left(p_{k} \in \mathcal{H}\left(p_{i}, p_{l}, 2\right) \mid \mathbf{x}_{i, l}=x\right) \leqslant \frac{3 x+8}{R^{3}}$. Since $\frac{n}{R^{3}}=\mu$, this amounts to dividing the terms over which we integrate by $\mu(3 x+8)$ which does not change the general shape of the integrals (a polynomial multiplied by an exponential) which are convergent. Notice that $B_{i}, B_{j}, B_{l}$ and $\omega$ now define a set of segments $t_{i} t_{l}$, rather than just a single segment. However, those segments define a closed 1-face only if none of them is occluded by one of the $n-3$ remaining balls. Any particular choice of a tangent $t_{i} t_{l}$ in the 1 -face will give a relevant cylinder $\mathcal{H}\left(t_{i}, t_{l}, 1\right)$ to use in the proofs.

Lemma 5.3. The expected number of 2-faces is $\Theta(n)$.

Proof. Since a 1-face has five incident 2-faces, the tight linear bound on the number of 1-faces gives a tight linear bound on the number of open 2-faces. The closed case is solved similarly to the proof of the upper bound in Theorem 2.1. We now consider $\left(\begin{array}{l}n \\ 2\end{array}\right)$ pairs of balls $B_{i}, B_{l}$ and we remove from the integrals the probability $\operatorname{Pr}\left(p_{j}, p_{k} \in \mathcal{H}\left(p_{i}, p_{l}, 2\right) \mid \mathbf{x}_{i, l}=x\right) \leqslant\left(\frac{3 x+8}{R^{3}}\right)^{2}$ which gives an $O(n)$ bound on the number of closed 2 -faces. $\square$

Lemma 5.4. The expected numbers of 3-faces and 4-faces are $\Theta(n)$.

Proof. A 3-face, corresponding to lines tangent to a ball, can only be closed if $n=1$. The number of open 3 -faces is linear by the fact that in general position a 2 -face is incident to four 3 -faces. The number of 4 -faces is linear since a 3 -face is incident to three 4 -faces.

6. Worst-case lower bound. We provide here a $\Omega\left(n^{2}\right)$ lower bound on the number of $k$-faces in the visibility complex. Recall that for the case of $n$ arbitrarily 
sized balls, Devillers and Ramos [6] presented a simple $\Omega\left(n^{3}\right)$ lower bound on the number of free segments tangent to 4 balls, which is also the number of vertices in the visibility complex. Their lower bound (see Figure 6.1) consists of (i) $\frac{n}{3}$ balls such that the view from the origin consists of $\frac{n}{3}$ disjoint disks centered on a circle, (ii) $\frac{n}{3}$ balls such that the view from the origin consists of $\frac{n}{3}$ disks whose boundaries are concentric circles intersecting (in projection) all the disks of (i), and (iii) $\frac{n}{3}$ tiny balls centered around the origin such that from any point on these $\frac{n}{3}$ tiny balls the view of the balls in (i) and (ii) is topologically invariant. Note that finding a $\Omega\left(n^{3}\right)$ lower bound on the number of free segments tangent to 4 balls, amongst $n$ balls of bounded radii, is to the best of our knowledge, open.

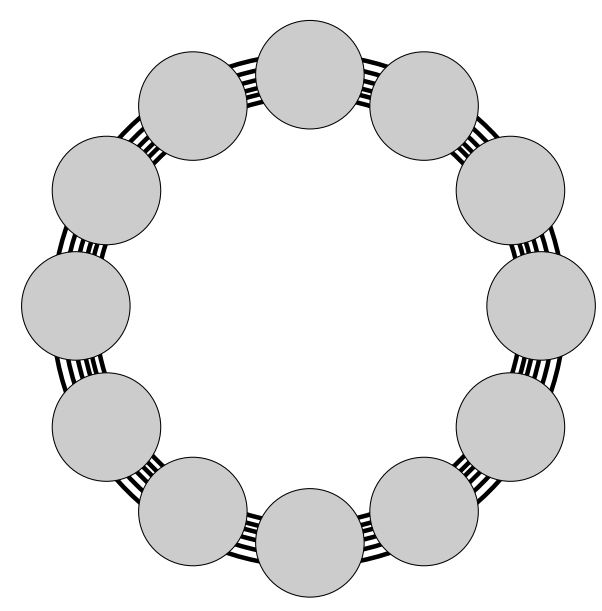

Fig. 6.1. Quadratic view from the origin [6].

Proposition 6.1. The number of $k$-faces in the visibility complex of $n$ disjoint unit balls in $\mathbb{R}^{3}$ is $\Omega\left(n^{2}\right)$ for all $k$ between 0 and 4 .

Proof. We first observe that the size of the visibility complex of $n$ unit balls can trivially be quadratic by having the balls sparsely distributed in the space such that any pair of balls defines a closed 2-face.

Getting a quadratic number of free lines tangent to four balls amongst a set of $n$ unit balls can be done by taking balls $B_{i}$ centered at $(2 i, 0,0)$ for $1 \leqslant i \leqslant \frac{n}{2}$ and balls $B_{j}^{\prime}$ centered at $(2 j, 10,0)$ for $1 \leqslant j \leqslant \frac{n}{2}$. Then, for any $i$ and $j$, the line through the points $(2 i+1,0,1)$ and $(2 j+1,10,1)$ is free and can be moved down so that it comes into contact with the four balls $B_{i}, B_{i+1}, B_{j}^{\prime}$ and $B_{j+1}^{\prime}$. This argument proves that the number of $k$-faces, for $0 \leqslant k \leqslant 2$, can be quadratic.

The free segment $(2 i, 1,0)(2 j, 9,0)$ belongs to the 4 -face consisting of maximal free segments with endpoints on $B_{i}$ and $B_{j}^{\prime}$. Thus there is a quadratic number of 4 -faces. The bound also applies to 3 -faces by considering lines tangent to $B_{i}$ and stabbing $B_{j}^{\prime}$.

In the above construction, the balls can be pushed together (they will intersect) so that they fit inside a spherical universe of radius $\sqrt[3]{n / \mu}$ without changing the result. Note also that the above construction can be slightly perturbed to obtain the same result for a set of $n$ unit balls, disjoint or not, with no 4 centers coplanar.

7. Generalizations. In this section we provide several generalizations of our results. 
7.1. Poisson distribution. Consider a set of unit balls whose centers are drawn by a 3-D Poisson point process of parameter $\mu$ in the universe $\mathcal{U}$. By a Poisson point process of parameter $\mu$ in $\mathcal{U}$ [12], we mean that we generate $X$ random points inside $\mathcal{U}$ so that

$$
\operatorname{Pr}(X=k)=\frac{(\mu \cdot \operatorname{Volume}(\mathcal{U}))^{k} \cdot \exp (-\mu \cdot \operatorname{Volume}(\mathcal{U}))}{k !}
$$

and for any disjoint subsets $M$ and $M^{\prime}$ of $\mathcal{U}$, the number of the points inside $M$ and the number of points inside $M^{\prime}$ are independent random variables. Note that Equation (7.1) yields that the expected number of points inside $\mathcal{U}$ is $\mu \cdot \operatorname{Volume}(\mathcal{U})=$ $\frac{4 \pi}{3} n$.

The following simple argument shows that our results extend to this distribution. Let $X$ be the random variable representing the number of centers of unit balls generated by a Poisson point process with parameter $\mu$ in $\mathcal{U}$, and let $Y$ be the random variable representing the number of $T 4$-segments amongst those balls. The expected number of $T 4$-segments is

$$
E(Y)=\sum_{k=0}^{\infty} E(Y \mid X=k) \cdot \operatorname{Pr}(X=k) .
$$

Theorem 2.1 gives $E(Y \mid X=k)=\Theta(k)$ and

$$
\operatorname{Pr}(X=k)=\frac{\left(\frac{4}{3} \pi n\right)^{k} \cdot \exp \left(-\frac{4}{3} \pi n\right)}{k !} .
$$

Thus

$$
\begin{aligned}
E(Y) & =\Theta\left(\frac{4}{3} \pi n \exp \left(-\frac{4}{3} \pi n\right) \sum_{k=1}^{\infty} \frac{\left(\frac{4}{3} \pi n\right)^{k-1}}{(k-1) !}\right) \\
& =\Theta\left(n \exp \left(-\frac{4}{3} \pi n\right) \exp \left(\frac{4}{3} \pi n\right)\right)=\Theta(n) .
\end{aligned}
$$

Therefore the expected number of $T 4$-segments amongst $n$ balls whose centers are generated by a Poisson point process with parameter $\mu$ in $\mathcal{U}$ is $\Theta(n)$. Similarly this bound extends to the expected size of the visibility complex.

We now investigate various models in which we change the shape of the universe or the nature of the objects.

7.2. Smooth convex universe. Our results can be generalized to the case where the universe is no longer a ball, but a homothet of a smooth convex set with homothety factor proportional to $\sqrt[3]{n}$. This can be achieved by considering the radius of curvature of the boundary of the universe, instead of $R$, in the proofs of the lemmas dealing with tangents outside the universe.

7.3. Other objects. Let $r_{\min }$ and $r_{\max }$ be two strictly positive real constants. In the following, we bound the expected number of $T 4$-segments amongst balls whose radii vary in the interval $\left[r_{\min }, r_{\max }\right]$, amongst polyhedra each enclosed between two concentric balls of radii $r_{\min }$ and $r_{\max }$, and amongst polygons each enclosed between two concentric circles of radii $r_{\min }$ and $r_{\max }$. The centers of the concentric balls or circles are called the centers of the polyhedra or polygons, respectively. In each case a $T 4$-segment is called outer if the centers of the two extremal objects it is tangent 
to are farther apart than $6 r_{\max }$ and are both at distance less than $2 r_{\max }$ from the boundary of $\mathcal{U}$. Otherwise the $T 4$-segment is called inner.

For these models, the proof of the $\Omega(n)$ lower bound on the expected number of T4-segments (Section 4) generalizes directly because, for the kind of objects we consider, there always exist placements of four of them such that they admit at least one common tangent line with multiplicity one.

7.3.1. Balls of various radii. We have considered a model where all the balls have the same radius. If we allow the radii to vary in the interval $\left[r_{\min }, r_{\max }\right]$, then the proof of the linear upper bound on the expected number of inner $T 4$-segments generalizes almost immediately by considering the volumes $\mathcal{H}\left(p_{i}, p_{l}, 2 r_{\text {max }}\right)$ and $\mathcal{H}\left(t_{i}, t_{l}, r_{\text {min }}\right)$ instead of $\mathcal{H}\left(p_{i}, p_{l}, 2\right)$ and $\mathcal{H}\left(t_{i}, t_{l}, 1\right)$.

Section 3.2.1 generalizes immediately to prove that the expected number of $T 4$ segments tangent to four balls $B_{i}, B_{j}, B_{k}$ and $B_{l}$ in that order such that $p_{i}$ and $p_{l}$ are closer to one another than $6 r_{\max }$ is $O(n)$. The only difficult task for extending Section 3.2.2 is the proof of the following analog of Lemma 3.8.

Lemma 7.1. When $\mathbf{x}_{i, l} \geqslant 6 r_{\max }, \mathbf{y}_{i} \geqslant 2 r_{\max }$ and $\mathcal{L}_{i, j, k, l}^{\omega}$ occurs, the volume of $\mathcal{H}\left(t_{i}, t_{l}, r_{\text {min }}\right) \cap \mathcal{U}$ is greater than $\frac{\pi}{24} r_{\text {min }}^{2}\left(\mathbf{x}_{i, l}-6 r_{\text {max }}\right)$.

Proof. The proof is similar to the proof of Lemma 3.8. Refer to Figure 7.1. Let $m$ be the midpoint of segment $t_{i} t_{l}$ and $K$ be the sphere of diameter $r_{m i n}$ centered on the point $c$ lying on segment $t_{i} p_{i}$ at distance $\frac{1}{2} r_{\min }$ from $t_{i}$. The sphere $K$ is entirely inside $\mathcal{H}\left(t_{i}, t_{l}, r_{\text {min }}\right) \cap \mathcal{U}, m$ lies in $\mathcal{H}\left(t_{i}, t_{l}, r_{\text {min }}\right)$ and a straightforward computation shows that $m$ is in $\mathcal{U}$ since $t_{i}$ is in $\mathcal{U}$ at distance at least $r_{\text {max }}$ from its boundary and $t_{l}$ is at distance at most $r_{\text {max }}$ from $\mathcal{U}$. Thus $\mathcal{H}\left(t_{i}, t_{l}, r_{\text {min }}\right) \cap \mathcal{U}$ contains the convex hull of $K$ and $m$ which contains the cone of apex $m$, of base a disk whose boundary is a great circle of $K$, and of height the distance from $m$ to the center $c$ of $K$. Now

$$
\begin{aligned}
\mathbf{x}_{i, l}=\left|p_{i} p_{l}\right| & \leqslant\left|p_{i} c\right|+|c m|+\left|m t_{l}\right|+\left|t_{l} p_{l}\right| \\
& \leqslant r_{\text {max }}+|c m|+\frac{1}{2}\left|t_{i} t_{l}\right|+r_{\text {max }} \\
& \leqslant 2 r_{\text {max }}+|c m|+\frac{1}{2}\left(\mathbf{x}_{i, l}+2 r_{\text {max }}\right) .
\end{aligned}
$$

Thus $|c m| \geqslant \frac{1}{2} \mathbf{x}_{i, l}-3 r_{\max }$ and the volume of the cone is at least $\frac{1}{3} \pi\left(\frac{r_{\min }}{2}\right)^{2}\left(\frac{1}{2} \mathbf{x}_{i, l}-\right.$ $\left.3 r_{\max }\right)=\frac{\pi}{24} r_{\min }^{2}\left(\mathbf{x}_{i, l}-6 r_{\max }\right)$.

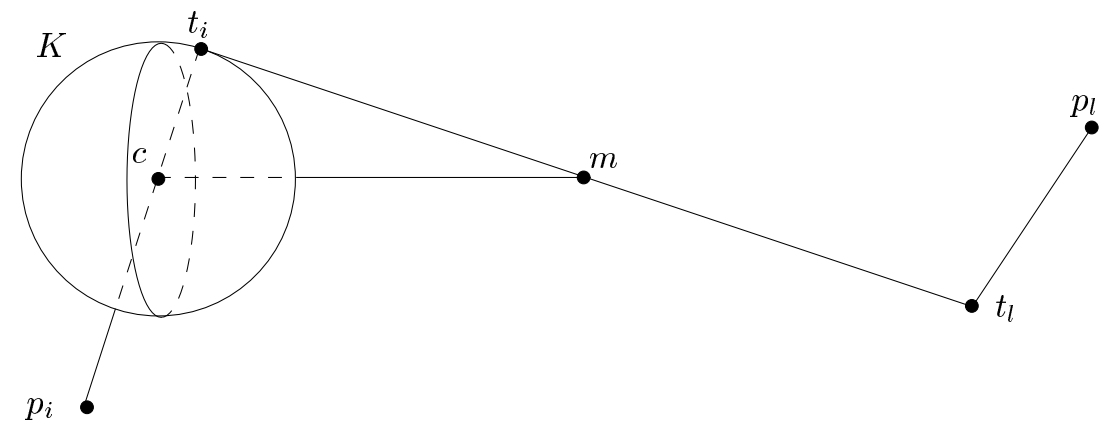

Fig. 7.1. For the proof of Lemma 7.1.

The rest of Section 3.2.2 generalizes easily for proving that the expected number of $T 4$-segments tangent to four balls $B_{i}, B_{j}, B_{k}$ and $B_{l}$ in that order such that $p_{i}$ 
and $p_{l}$ are farther apart than $6 r_{\max }$ and $p_{i}$ is farther than $2 r_{\max }$ from the boundary of $\mathcal{U}$, is $O(n)$. Hence the expected number of inner T4-segments is $O(n)$.

Our proof cannot be extended to provide a linear upper bound on the expected number of outer T4-segments. This is because, if balls $B_{i}$ and $B_{l}$ are of radius $r_{\max }$ then a line segment $t_{i} t_{l}$ tangent to $B_{i}$ and $B_{l}$ might be outside $\mathcal{U}$ and at distance greater than $r_{\text {min }}$ from its boundary. Then $\mathcal{H}\left(t_{i}, t_{l}, r_{\text {min }}\right)$ does not intersect $\mathcal{U}$ and we cannot bound $\mathcal{H}\left(t_{i}, t_{l}, r_{\text {min }}\right) \cap \mathcal{U}$ from below by a positive constant as in Lemma 3.12, which is crucial for the proof of Lemma 3.13 and thus for Proposition 3.18.

However, by not taking into account the occlusion in the proof of Proposition 3.18, we get that the expected number of outer $T 4$-segments is $O\left(n^{2}\right)$. Refer to the proof of Proposition 3.18 and consider $I_{x>6 r_{\max }, y<2 r_{\max }}$, the analog of $I_{x>6, y<1}$ for this case. The analogs of Lemmas 3.4 and 3.5 yield that

$$
\operatorname{Pr}\left(\delta_{i, j, k, l}^{\omega} \mid \mathbf{x}_{i, l}=x, \mathbf{y}_{i}=y, \mathbf{y}_{l} \leqslant \mathbf{y}_{i}\right) \leqslant \frac{\left(3 x r_{\max }^{2}+8 r_{\max }^{3}\right)^{2}}{R^{6}} .
$$

Lemma 3.16 still holds and we can easily prove the analog of Lemma 3.17. Both results imply that

$$
\begin{aligned}
I_{x>6 r_{\max }, y<2 r_{\max }} & \leqslant \int_{x=6 r_{\max }}^{2 R} \int_{y=0}^{2 r_{\max }} \frac{\left(3 x r_{\max }^{2}+8 r_{\max }^{3}\right)^{2}}{R^{6}} \cdot \frac{6 x y d x}{R^{3}} \cdot \frac{3 d y}{R} \\
& \in O\left(\frac{1}{R^{6}}\right)=O\left(\frac{1}{n^{2}}\right) .
\end{aligned}
$$

Hence the expected number of inner $T 4$-segments is $O(n)$ and the expected number of outer T4-segments is $O\left(n^{2}\right)$. This still improves the result of Durand et al. [11] who proved a bound of $O\left(n^{8 / 3}\right)$ for the same model.

In this section we have assumed that the sphere centers are uniformly distributed but we have made no assumption on the distribution of the radii of the spheres in the interval $\left[r_{\min }, r_{\max }\right]$, which are thus assumed to be worst case. The addition of some hypothesis on the radii distribution may yield better results on the number of outer T4-segments.

7.3.2. Polyhedra of bounded aspect ratio. Consider polyhedra of constant complexity, each enclosed between two concentric balls of radii $r_{\min }$ and $r_{\max }$ whose centers are uniformly distributed in $\mathcal{U}$. In such a case, as for balls of various radii, the $O(n)$ bound on the expected number of inner $T 4$-segments immediately applies as well as the $O\left(n^{2}\right)$ bound on the expected number of outer T4-segments.

7.3.3. Polygons of bounded aspect ratio. Our proof technique can also be generalized to non-fat 3D objects such as polygons. Consider polygons of constant complexity enclosed between two coplanar concentric circles of radii $r_{\min }$ and $r_{\max }$, and whose centers and normals are independently chosen from the uniform distributions over $\mathbb{R}^{3}$ and $\mathbb{S}^{2}$. Let $T_{1}, \ldots, T_{n}$ be such polygons with respective normals $\mathbf{n}_{1}, \ldots, \mathbf{n}_{n}$ and centers $p_{1}, \ldots, p_{n}$.

Four polygons $T_{i}, T_{j}, T_{k}$ and $T_{l}$ have a common tangent line that meet them in that order only if $p_{j}$ and $p_{k}$ lie in $\mathcal{H}\left(p_{i}, p_{l}, 2 r_{\max }\right)$. This implies, as in Section 3.2.1, that the expected number of $T 4$-segments tangent to four polygons $T_{i}, T_{j}, T_{k}$ and $T_{l}$ in that order such that $p_{i}$ and $p_{l}$ are closer to one another than some constant, say $6 r_{\max }$, is $O(n)$.

When such a tangent, denoted $t_{i} t_{l}$, exists, it is not occluded only if, for any $\gamma \neq i, j, k, l$, point $p_{\gamma}$ does not lie in the interior of $\mathcal{H}\left(t_{i}, t_{l}, r_{\min } \cos \theta_{\gamma}\right)$ where $\theta_{\gamma}$ 


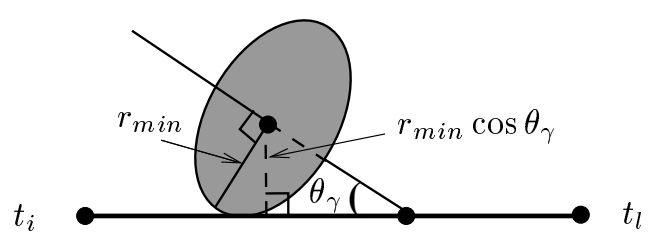

(a)

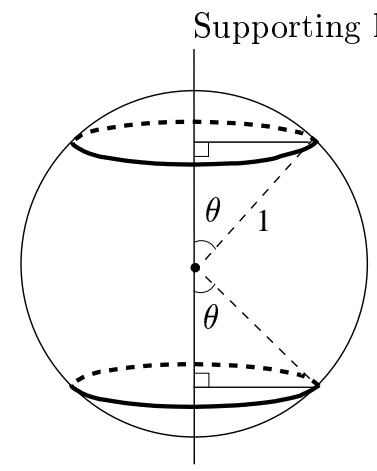

(b)

Fig. 7.2. Illustration for the case of polygons of bounded aspect ratio.

denotes the angle between $\mathbf{n}_{\gamma}$ and the supporting line of $t_{i} t_{l}$ (see Figure 7.2 (a) and Lemma 3.3). Let $\gamma$ be an integer distinct from $i, j, k$ and $l$. By the Total Probability Theorem, the probability that $T_{\gamma}$ does not occlude the tangent line segment $t_{i} t_{l}$ is bounded from above by

$$
\int_{\theta=0}^{\pi / 2} \operatorname{Pr}\left(p_{\gamma} \notin \mathcal{H}\left(t_{i}, t_{l}, r_{\min } \cos \theta_{\gamma}\right) \mid \theta_{\gamma}=\theta\right) \cdot \operatorname{Pr}\left(\theta \leqslant \theta_{\gamma}<\theta+d \theta\right) .
$$

Similarly as in Lemma 7.1, when the tangent $t_{i} t_{l}$ exists, $\mathbf{x}_{i, l} \geqslant 6 r_{\max }$ and $\mathbf{y}_{i} \geqslant 2 r_{\max }$, the volume of $\mathcal{H}\left(t_{i}, t_{l}, r_{\text {min }}\right) \cap \mathcal{U}$ is greater than $\frac{\pi}{24}\left(r_{\text {min }} \cos \theta_{\gamma}\right)^{2}\left(\mathbf{x}_{i, l}-6 r_{\text {max }}\right)$. Thus

$$
\operatorname{Pr}\left(p_{\gamma} \notin \mathcal{H}\left(t_{i}, t_{l}, r_{\text {min }} \cos \theta_{\gamma}\right) \mid \theta_{\gamma}=\theta\right) \leqslant 1-\frac{\left(r_{\min } \cos \theta_{\gamma}\right)^{2}\left(\mathbf{x}_{i, l}-6 r_{\max }\right)}{32 R^{3}} .
$$

The probability that $\theta_{\gamma}$ is in between $\theta$ and $\theta+d \theta$ is $\sin \theta d \theta$, which corresponds to twice the area of the spherical shell between the latitudes $\theta$ and $\theta+d \theta$ on the unit sphere, divided by the area of the unit sphere (see Figure $7.2(\mathrm{~b})$ ). Thus when $p_{i}$ is at distance greater than $6 r_{\max }$ from $p_{l}$ and at distance greater than $2 r_{\max }$ from the boundary of $\mathcal{U}$, the probability that $T_{\gamma}$ does not occlude the tangent line segment $t_{i} t_{l}$ is bounded from above by

$$
\int_{\theta=0}^{\pi / 2}\left(1-\frac{\left(r_{\min } \cos \theta_{\gamma}\right)^{2}\left(\mathbf{x}_{i, l}-6 r_{\max }\right)}{32 R^{3}}\right) \sin \theta d \theta=1-\frac{r_{\min }^{2}\left(\mathbf{x}_{i, l}-6 r_{\max }\right)}{96 R^{3}} .
$$

Then, similarly as in Lemma 3.9 , the probability that the tangent line segment $t_{i} t_{l}$ is not occluded, when $p_{i}$ is at distance greater than $6 r_{\max }$ from $p_{l}$ and at distance greater than $2 r_{\max }$ from the boundary of $\mathcal{U}$, is at most

$$
55 \exp \left(-\frac{\mu r_{\min }^{2}\left(\mathbf{x}_{i, l}-6 r_{\max }\right)}{96}\right) \text {. }
$$

We thus get the analog of Proposition 3.10 for the model considered here which implies that the expected number of $T 4$-segments tangent to four polygons $T_{i}, T_{j}, T_{k}$ and $T_{l}$ in that order such that $p_{i}$ and $p_{l}$ are farther apart than $6 r_{\text {max }}$ and $p_{i}$ is farther than $2 r_{\text {max }}$ from the boundary of $\mathcal{U}$ is $O(n)$.

We thus get that the expected number of inner T4-segments is $O(n)$. Moreover, as for balls of various radii, the expected number of outer T4-segments is $O\left(n^{2}\right)$. 
8. Conclusion. In this paper, we proved that the expected number of $T 4$ segments amongst $n$ uniformly distributed unit balls in $\mathbb{R}^{3}$ is $\Theta(n)$. We also proved that the expected size of the visibility complex of $n$ uniformly distributed unit balls is $\Theta(n)$. Equivalently the expected number of combinatorially different visibility events amongst $n$ uniformly distributed unit balls is $\Theta(n)$. We then proved that $\Theta(n)$ also bounds the expected number of $T 4$-segments occurring not too close to the boundary of the universe for various other models such as $n$ uniformly distributed polyhedra, or polygons, of bounded aspect ratio and constant complexity. For these models, we also provided a $O\left(n^{2}\right)$ bound on the expected number of all the T4-segments.

This paper is an attempt to analyze the average-case behavior of the size of visibility structures. The distribution models of scene objects investigated here are theoretical in nature since objects in graphics scenes are seldom distributed uniformly or by a Poisson process. However, our results are important in a context where there are few rigorous results either theoretical or experimental. They provide theoretical ground to support the empirical evidence indicating that the worst-case upper bound on the number of visibility events is largely pessimistic in practical situations. As a consequence, there is reason to believe that an output-sensitive algorithm for computing all visibility events may work in practice.

Practitioners will be concerned about the size of the constant hidden in the $\Theta$ notation. We have calculated (in the proofs of Section 3) this constant to be no larger than $2^{16} \mu^{3}+2^{31} \mu+2^{37} e^{-\mu / 3}\left(\mu^{2}+1 / \mu^{2}\right)$. Of course this is shocking. We suppose that the constant is actually much smaller. However estimating it in practice is a difficult problem which is still to be solved. After solving this problem, an interesting experiment will be to compare the number of visibility events occurring in a realistic graphic scene with the theoretical bound for uniformly distributed objects.

The results proved here also provide new insight on the complexity of other visibility structures. Consider for instance the aspect graph, a partition of viewpoint space into maximal connected regions by surfaces along which visibility events are observed. As explained in [21], the complexity of the aspect graph is dominated by $\delta^{m}$, where $\delta$ is the degree of the surface corresponding to lines "tangent" to 3 objects and $m$ the dimension of the viewpoint space. For a scene composed of $n$ disjoint spheres, $\delta$ is trivially $O\left(n^{3}\right)$, so the aspect graph has $O\left(n^{6}\right)$ orthographic views and $O\left(n^{9}\right)$ perspective views. However the results of this paper show that the expected value of $\delta$ is $\Theta(n)$ since the expected number of families of lines tangent to three objects (related to the 1-faces of the visibility complex) is linear and the degree of each family is bounded. It would thus be interesting to get a good bound on the expected value of $\delta^{2}$ and $\delta^{3}$ which is related to bounding the expected value of the square and the cube of the number of combinatorially different visibility events. Note that the former would also give the standard deviation of the expected number of combinatorially different visibility events. Similar observations hold for the polyhedral case.

Appendix A. Volume of the intersection of a 3D hippodrome with a ball. Recall that $\mathcal{U}$ is a ball of radius $R$ centered at $O$. Let $B_{i}$ and $B_{l}$ be two unit balls whose centers $p_{i}$ and $p_{l}$ are in $\mathcal{U}$, within distance 1 from its boundary, and distance $x \geqslant 6$ apart. Let $t_{i} t_{l}$ be a line segment tangent to $B_{i}$ and $B_{l}$ at its endpoints. The section is devoted to the proof of the following proposition which leads directly to Lemma 3.12 .

Proposition A.1. For any $0 \leqslant s \leqslant 1$ such that segment $t_{i} t_{l}$ is at distance less than $R+1-s$ from $O$, the volume of $\mathcal{H}\left(t_{i}, t_{l}, 1\right) \cap \mathcal{U}$ is larger than $\frac{1}{6 \sqrt{2}}(x-5) s \sqrt{s}$.

We proceed as follows. Let $v$ be such that the distance from $O$ to the segment 


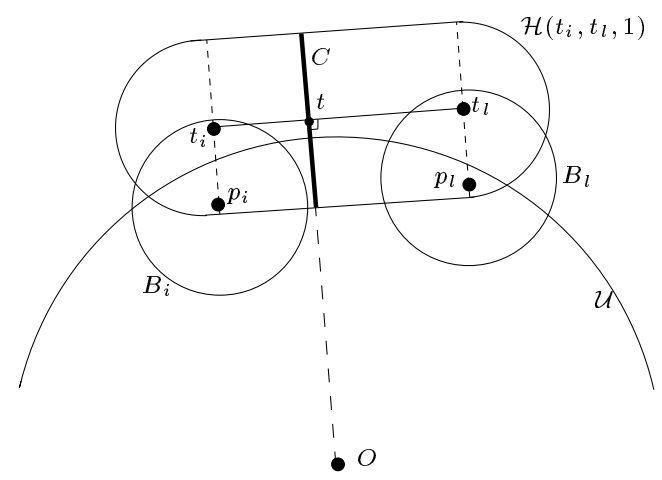

(a) $t$ is not equal to $t_{i}$ or $t_{l} ; \theta=0$.

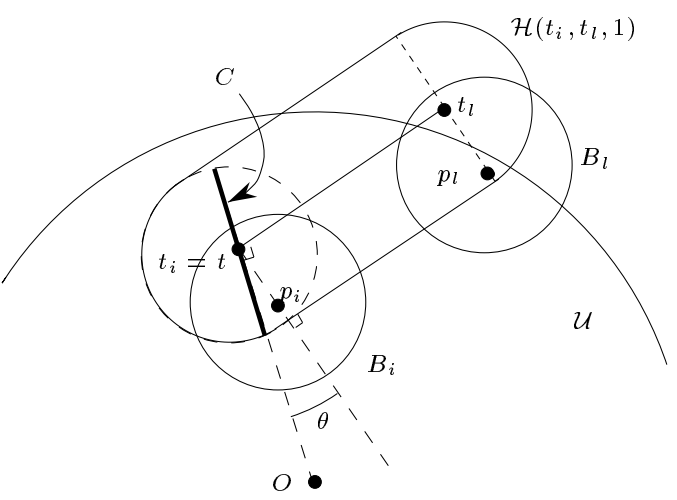

(b) $t$ is equal to $t_{i}$.

Fig. A.1. For the definition of $t$ and $C$ ( $C$ is shown from the side view).

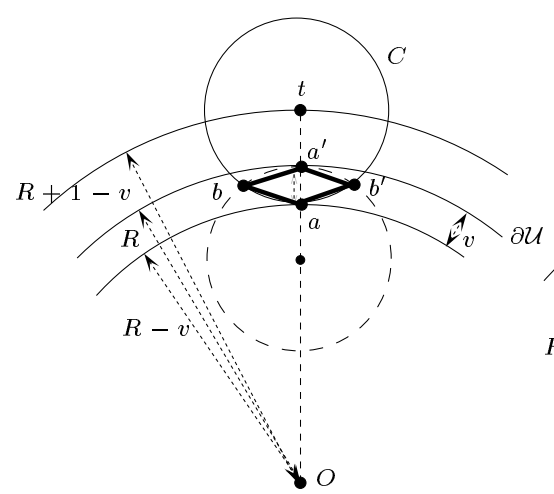

(a) $v \in[0,1]$

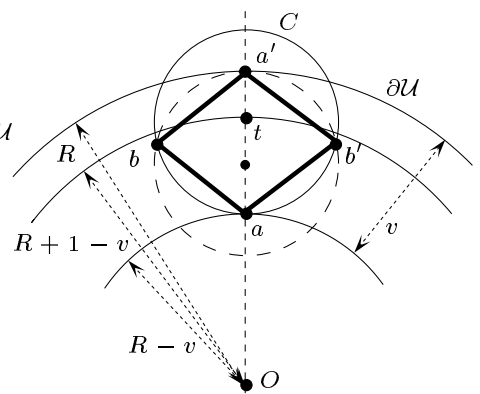

(b) $v^{\prime} \in[1,2]$

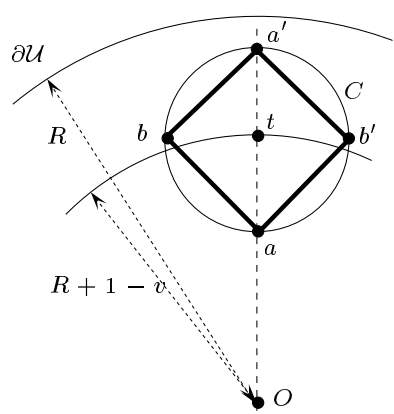

(c) $v>2$

Fig. A.2. For the definition of $a, a^{\prime}, b, b^{\prime}$.

$t_{i} t_{l}$ is $R+1-v$, and let $t$ be the point on segment $t_{i} t_{l}$ closest to $O$ (see Figure A.1). Assume without loss of generality that $t$ is closer to $t_{i}$ than to $t_{l}$. Let $C$ (resp. $D$ ) be the unit radius circle (resp. disk) centered at $t$ in the plane, denoted $\mathcal{P}$, containing the vectors $\overrightarrow{O t}$ and the cross product of $\overrightarrow{O t}$ and ${\overrightarrow{t_{i}}}_{l}$. Let $\theta$ be the angle between the plane orthogonal to $t_{i} t_{l}$ and $\mathcal{P}$. We first prove the following lemma.

Lemma A.2. The volume of $\mathcal{H}\left(t_{i}, t_{l}, 1\right) \cap \mathcal{U}$ is greater than

$$
\frac{1}{3} \min \left(2, \frac{v \sqrt{v}}{\sqrt{2}}\right) \cdot \min \left(\frac{x-2}{2},(x-2) \cos \theta-1\right) \text {. }
$$

Proof. Let $a$ denote the closest point on $C$ from $O, a^{\prime}$ the farthest point in $D \cap \mathcal{U}$ from $O$, and $b$ and $b^{\prime}$ the two points of intersection of $C$ and the perpendicular bisector of segment $a a^{\prime}$ (see Figure A.2).

The volume of $\mathcal{H}\left(t_{i}, t_{l}, 1\right) \cap \mathcal{U}$ is greater than the volume of the convex hull of $a, b, a^{\prime}, b^{\prime}$ and $p_{l}$ because $\mathcal{H}\left(t_{i}, t_{l}, 1\right) \cap \mathcal{U}$ is convex and contains these five points. The volume of this polyhedron is equal to one third of the area of its base, the quadrilateral with vertices $a, b, a^{\prime}, b^{\prime}$, times its height, the distance from $p_{l}$ to the plane $\mathcal{P}$ containing 
$a, b, a^{\prime}, b^{\prime}$.

We first compute a lower bound on the area of the quadrilateral with vertices $a, b, a^{\prime}, b^{\prime}$. If $v \leqslant 2$ (see Figure A.2 (a) and (b)), then the length of $a a^{\prime}$ is equal to $v$, and a simple calculation gives that the length of $b b^{\prime}$ is equal to $2 \sqrt{v-\frac{v^{2}}{4}} \geqslant \sqrt{2 v}$. Thus the area of the quadrilateral $a, b, a^{\prime}, b^{\prime}$ is greater than $\frac{v \sqrt{v}}{\sqrt{2}}$. If $v>2$ (see Figure A.2 (c)), then $C$ is entirely contained in $\mathcal{U}$ and the area of the quadrilateral $a, b, a^{\prime}, b^{\prime}$ is equal to 2 . Thus, the area of the quadrilateral is at least $\min \left(2, \frac{v \sqrt{v}}{\sqrt{2}}\right)$.

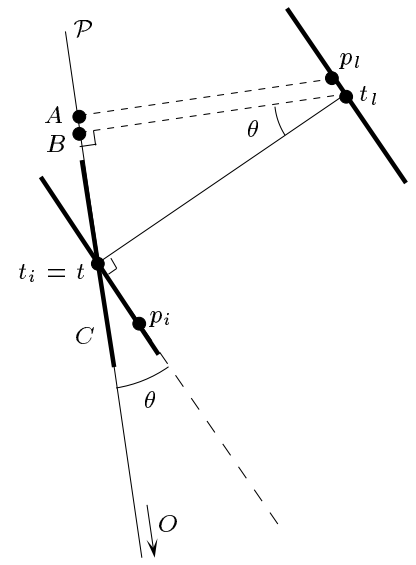

Fig. A.3. The height from $p_{l}$ to $\mathcal{P}$ is greater than $\left|t_{i} t_{l}\right| \cos \theta-1$.

The volume of the polyhedron is thus greater than $\frac{1}{3} \min \left(2, \frac{v \sqrt{v}}{\sqrt{2}}\right)$ times the distance from $p_{l}$ to the plane $\mathcal{P}$. We consider two cases.

First, suppose that $t$ belongs to the interior of the segment $t_{i} t_{l}$ (see Figure A.1 (a)). Then, the height is equal to the distance from $t_{l}$ to $t$ since $p_{l}$ and $a, b, a^{\prime}, b^{\prime}$ belong, respectively, to the two planes, orthogonal to $t_{i} t_{l}$ and passing through $t_{l}$ and $t$, respectively. Since $t_{i}$ and $t_{l}$ belong to $B_{i}$ and $B_{l}$, they are at least distance $x-2$ apart, thus $t$ and $t_{l}$ are at least distance $\frac{x-2}{2}$ apart. Thus, the height from $p_{l}$ to $\mathcal{P}$ is at least $\frac{x-2}{2}$.

Second, suppose that $t=t_{i}$ (see Figure A.1 (b)); $t \neq t_{l}$ since we assumed that $t$ is closer to $t_{i}$ than to $t_{l}$. Refer to Figure A.3. Let $A$ and $B$ be the orthogonal projections of $p_{l}$ and $t_{l}$ onto $\mathcal{P}$, respectively. Note that the lengths of $A p_{l}$ and $B t_{l}$ are the distances from $p_{l}$ and $t_{l}$ to the plane $\mathcal{P}$, respectively.

Considering the triangle $\triangle A p_{l} t_{l}$ and that the distance between $t_{l}$ and $p_{l}$ is 1 , we obtain that $\left|A p_{l}\right| \geqslant\left|A t_{l}\right|-\left|t_{l} p_{l}\right|=\left|A t_{l}\right|-1$, where $|a b|$ denotes the length of segment $a b$. Since $A \in \mathcal{P}$ and the length of $B t_{l}$ is the distance from $t_{l}$ to the plane $\mathcal{P}$, the length of $A t_{l}$ is greater than that of $B t_{l}$, thus $\left|A p_{l}\right| \geqslant\left|B t_{l}\right|-1$.

To bound the length of $B t_{l}$, we now consider the triangle $\triangle B t_{l} t$. The angle $\angle B t_{l} t$ is the angle between the normal of the plane $\mathcal{P}$ and $t_{i} t_{l}$, that is, by definition, $\theta$. So the length of $B t_{l}$ is the length of $t_{i} t_{l}$ times $\cos \theta$ and, since $\left|t_{i} t_{l}\right|$ is at least $x-2,\left|B t_{l}\right|$ is greater than $(x-2) \cos \theta$. Thus the length of $A p_{l}$ is greater than $(x-2) \cos \theta-1$.

Hence the distance from $p_{l}$ to the plane $\mathcal{P}$ is greater than $\min \left(\frac{x-2}{2},(x-2) \cos \theta-1\right)$ and thus the volume of $\mathcal{H}\left(t_{i}, t_{l}, 1\right) \cap \mathcal{U}$ is greater than $\frac{1}{3} \min \left(2, \frac{v \sqrt{v}}{\sqrt{2}}\right) \cdot \min \left(\frac{x-2}{2},(x-\right.$ 2) $\cos \theta-1)$.

The following lemma bounds $\cos \theta$. 


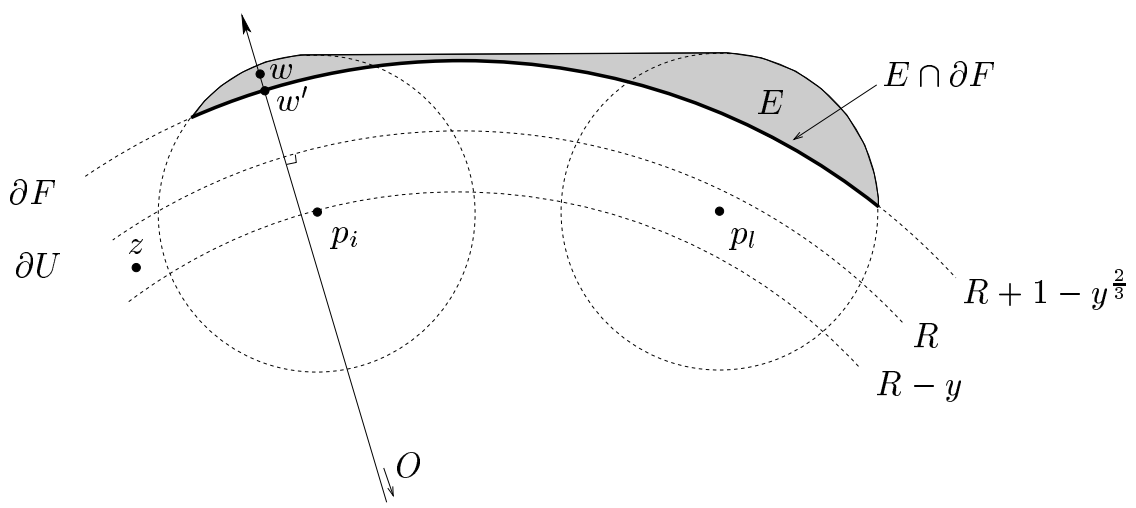

Fig. B.1. The part $E$ of $\mathcal{H}\left(p_{i}, p_{l}, 1\right)$ outside $F$.

LEMma A.3. The angle $\theta$ is such that $\cos \theta \geqslant \frac{\sqrt{7}}{4}$.

Proof. Note first that this lemma is intuitively obvious. Indeed (see Figure A.1 (b)), if $x$ is sufficiently large and if $t_{i}$ is the closest point on segment $t_{i} t_{l}$ to $O$, then the angle between the plane supporting $C$ and the segment $t_{i} t_{l}$ is necessarily close to $\pi / 2$, which implies that $\theta$ is close to 0 . We now prove the lemma.

Refer to Figure A.3 and consider the triangle $\triangle O t_{i} t_{l}$. Let $|a b|$ denote the length of segment $a b$. Then the law of cosines yields

$$
\begin{aligned}
\left|O t_{l}\right|^{2} & =\left|O t_{i}\right|^{2}+\left|t_{i} t_{l}\right|^{2}-2 \cdot\left|O t_{i}\right| \cdot\left|t_{i} t_{l}\right| \cdot \cos \left(\frac{\pi}{2}+\theta\right) \\
& =\left|O t_{i}\right|^{2}+\left|t_{i} t_{l}\right|^{2}+2 \cdot\left|O t_{i}\right| \cdot\left|t_{i} t_{l}\right| \cdot \sin \theta
\end{aligned}
$$

which gives that

$$
\sin \theta=\frac{\left|O t_{l}\right|^{2}-\left|O t_{i}\right|^{2}-\left|t_{i} t_{l}\right|^{2}}{2 \cdot\left|O t_{i}\right| \cdot\left|t_{i} t_{l}\right|} .
$$

The centers $p_{i}$ and $p_{l}$ of balls $B_{i}$ and $B_{l}$ are distance $x \geqslant 6$ apart and at distance less than 1 from the boundary of $\mathcal{U}$, so $\left|t_{i} t_{l}\right| \geqslant 4,\left|O t_{i}\right| \geqslant R-2$ and $\left|O t_{l}\right| \leqslant R+1$. Hence

$$
\sin \theta \leqslant \frac{(R+1)^{2}-(R-2)^{2}-4^{2}}{2 \cdot(R-2) \cdot 4} \leqslant \frac{6(R-2)}{8(R-2)}=\frac{3}{4} .
$$

Using $\cos \theta=\sqrt{1-(\sin \theta)^{2}}$ proves that $\cos \theta \geqslant \frac{\sqrt{7}}{4}$. $\square$

We can now conclude the proof of Proposition A.1. For any $0 \leqslant s \leqslant 1$, if segment $t_{i} t_{l}$ is at distance $R+1-v \leqslant R+1-s$ from the center of $\mathcal{U}$, then $v \geqslant s$. By Lemma A.3, $(x-2) \cos \theta-1 \geqslant \frac{x-5}{2}$ which means that $\min \left(\frac{x-2}{2},(x-2) \cos \theta-\right.$ $1) \geqslant \frac{x-5}{2}$. Thus Lemma A.2 gives that the volume of $\mathcal{H}\left(t_{i}, t_{l}, 1\right) \cap \mathcal{U}$ is greater than $\frac{1}{6 \sqrt{2}}(x-5) \min (2 \sqrt{2}, v \sqrt{v}) \geqslant \frac{1}{6 \sqrt{2}}(x-5) \min (2 \sqrt{2}, s \sqrt{s})=\frac{1}{6 \sqrt{2}} s \sqrt{s}(x-5)$ since $s \leqslant 1$. Hence the volume of $\mathcal{H}\left(t_{i}, t_{l}, 1\right) \cap \mathcal{U}$ is greater than $\frac{1}{6 \sqrt{2}} s \sqrt{s}(x-5)$.

Appendix B. Volume of $K$. Recall that $\mathcal{U}$ is a ball of radius $R$ centered at $O$ and let $p_{i}$ and $p_{l}$ be two points in $\mathcal{U}$ within distance 1 of its boundary and distance $x$ apart. Let $y$ be a real number such that $0 \leqslant y<1$. Let $F$ be the open ball with center $O$ and radius $R+1-y^{\frac{2}{3}}$ and $\partial F$ its frontier. Let $E$ be the part of $\mathcal{H}\left(p_{i}, p_{l}, 1\right)$ that is outside $F$ and $K$ be the intersection of $\mathcal{U}$ with the union of all unit balls centered 
on points in $E$ (see Figure 3.3). This section is devoted to the proof of the following proposition used in the proof of Lemma 3.15 .

Proposition B.1. The volume of $K$ is bounded from above by $12 \pi^{2}(x+6) y$.

Lemma B.2. If $z \in \mathcal{U}$ is at distance less than 1 from $E$, then $z$ is at distance less than 1 from $E \cap \partial F$.

Proof. Let $z \in \mathcal{U}$ and $w \in E$ be two points at distance less than 1 and refer to Figure B.1. Let $w^{\prime}$ be the point of intersection of $\partial F$ and the ray from $O$ through $w$. For any ball $B$ centered in $\mathcal{U}, B \backslash F$ lies in the cone of center $O$ and base $B \cap \partial F$. Thus $E=\mathcal{H}\left(p_{i}, p_{l}, 1\right) \backslash F$ lies in the cone of center $O$ and base $E \cap \partial F$. Hence the ray from $O$ through $w$ lies in this cone and $w^{\prime} \in E \cap \partial F$. On the other hand, $\left|z w^{\prime}\right| \leqslant|z w|$ since $z \in F, w^{\prime} \in \partial F$ and $w$ lies outside $F$ on the ray from $O$ through $w^{\prime}$. Thus, since $w^{\prime} \in E \cap \partial F$ and $|z w|<1$ by hypothesis, the distance from $z$ to $E \cap \partial F$ is less than 1. $\square$

The above lemma implies that $K$ is the intersection of $\mathcal{U}$ with the union of all unit balls centered on $E \cap \partial F$. To bound the volume of $K$, we enclose $E \cap \partial F$ in a subset of $\partial F$ that will be easier to deal with.

Let $B(p)$ denote the ball of unit radius centered at $p$. Let $\pi(p)$ be the point that maximizes (under inclusion) the intersection $\partial F \cap B(q)$ for all $q$ on the ray from $O$ through $p$. A simple computation yields that the distance between $\pi(p)$ and $O$ is

$$
R_{y}=\sqrt{\left(R+1-y^{\frac{2}{3}}\right)^{2}-1}
$$

Thus $\pi$ is the orthogonal projection onto the sphere centered at $O$ of radius $R_{y}$. Now let $\pi^{\prime}(p)$ be the point that maximizes (under inclusion) the intersection $\partial F \cap B(q)$ for all $q$ on the radius of $\mathcal{U}$ through $p$ (that is the part inside $\mathcal{U}$ of the ray from $O$ through $p$ ). Similarly, $\pi^{\prime}$ is the orthogonal projection onto the sphere centered at $O$ of radius

$$
R^{\prime}=\min \left(R, R_{y}\right)
$$

Let $G$ be the union of the spherical caps $\partial F \cap B\left(\pi^{\prime}(p)\right)$ for all $p$ on the segment from $p_{i}$ to $p_{l}$ (see Figure B.2). Let $H$ denote the points of $\mathcal{U}$ at distance less than or equal to 1 from $G$ (see Figure B.3).

LEMMA B.3. $K \subseteq H$.

Proof. $E \cap \partial F$ is the union of $\partial F \cap B(p)$ for all $p$ on the segment $p_{i} p_{l}$. Furthermore, for any such $p, \partial F \cap B(p) \subseteq \partial F \cap B\left(\pi^{\prime}(p)\right)$ by definition of $\pi^{\prime}$ since $p \in \mathcal{U}$. Thus $E \cap \partial F$ is contained in $G$.

By Lemma B.2, $K$ is the intersection of $\mathcal{U}$ with the union of all unit balls centered on $E \cap \partial F$. Thus $K$ is contained in $H$, the union of all unit balls centered in $G$.

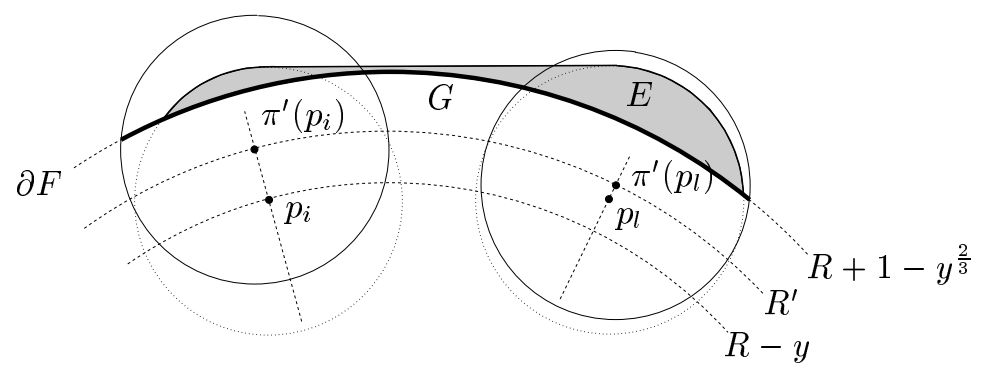

Fig. B.2. $G$, a part of $\partial F$ enclosing $E \cap \partial F$. 


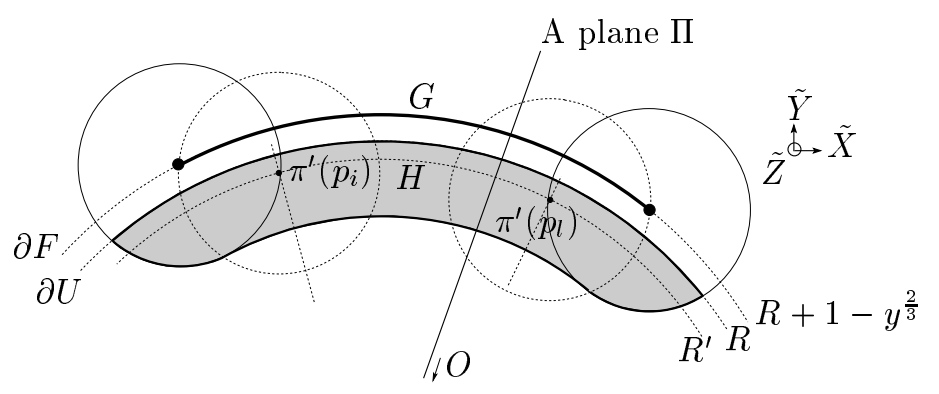

Fig. B.3. The region $H$ and a plane $\Pi$.

To bound the volume of $H$ from above, we first bound the area of its section by planes $\Pi$ that contain $O$ and are orthogonal to the plane, denoted $\left(O, p_{i}, p_{l}\right)$, containing $O, p_{i}$ and $p_{l}$ (see Figures B.3 and B.4).

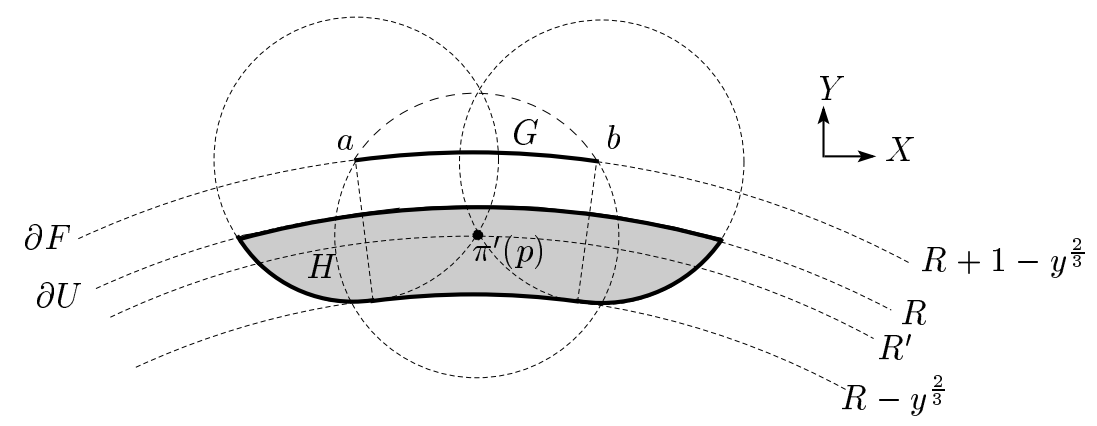

Fig. B.4. Section of $H$ by a plane $\Pi$ intersecting segment $p_{i} p_{l}$ at $p$.

Lemma B.4. The area of $\Pi \cap H$ is less than $12 \pi y$.

Proof. The section of $G$ by a plane $\Pi$ is a circular arc on $\partial F$. If $\Pi$ intersects the segment $p_{i} p_{l}$, let $p$ denote the point of intersection, then the circular arc is the intersection of $\partial F$ and the disk $B\left(\pi^{\prime}(p)\right) \cap \Pi$ (refer to Figures B.2 and B.4). Otherwise, the circular arc is the intersection of $\partial F$ and the disk $B\left(\pi^{\prime}\left(p_{i}\right)\right) \cap \Pi$ or $B\left(\pi^{\prime}\left(p_{l}\right)\right) \cap \Pi$ (see Figure B.2). The disk has radius 1 in the former case and radius less than one in the latter case. In both cases the center of the disk is at distance $R^{\prime}$ from $O$. Thus the length of the circular arc $G \cap \Pi$ is maximal if and only if $\Pi$ intersects the segment $p_{i} p_{l}$. Thus the area of $\Pi \cap H$ is maximal if and only if $\Pi$ intersects the segment $p_{i} p_{l}$. Hence we can assume that $\Pi$ is such a plane. Let $p$ denote its intersection with segment $p_{i} p_{l}$.

Let $a$ and $b$ denote the endpoints of $G \cap \Pi$ and refer to Figure B.4. Points $a$ and $b$ are the intersection of $\partial F$ and the circle in $\Pi$ of radius 1 centered at $\pi^{\prime}(p)$. The lines $(O a)$ and $(O b)$ split $\Pi \cap H$ into three parts, a left, a central and a right part. Symmetries with respect to the lines $(O a)$ and $(O b)$ send the left and right parts into the central one. Hence, the area of $\Pi \cap H$ is bounded by 3 times the area of its central part. This part is delimited by the two rays from $O$ through $a$ and $b$, and the two circles in $\Pi$ with center $O$ and radii $R$ and $R-y^{\frac{2}{3}}$. So, if $\alpha$ denotes the length of the circular arc $a b$, the area $A$ of the central part is

$$
A=\frac{\alpha}{2 \pi\left(R+1-y^{\frac{2}{3}}\right)} \cdot \pi\left(R^{2}-\left(R-y^{\frac{2}{3}}\right)^{2}\right)=\alpha \frac{2 R y^{\frac{2}{3}}-y^{\frac{4}{3}}}{2\left(R+1-y^{\frac{2}{3}}\right)} \leqslant \alpha y^{\frac{2}{3}} .
$$




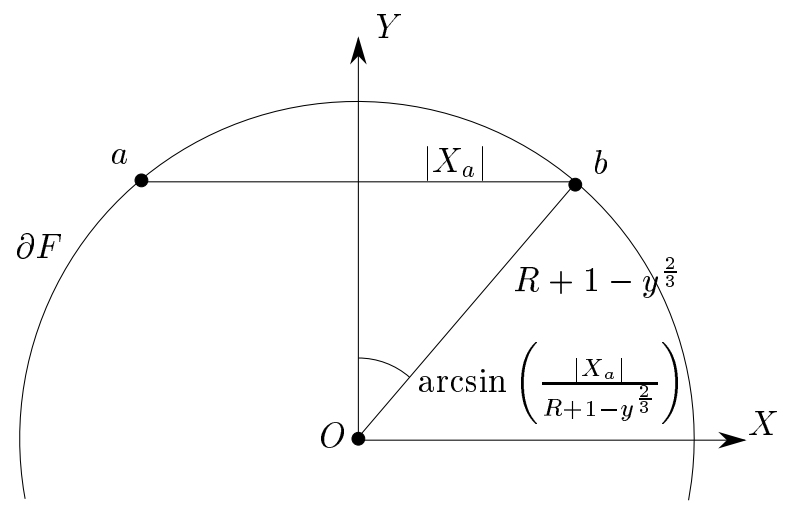

Fig. B.5. The length of the circular arcs $a b$.

We now bound the length $\alpha$ of the arc $a b$. We choose an orthonormal frame $\left(\pi^{\prime}(p), X, Y\right)$ in $\Pi$ such that $O$ has coordinates $\left(0,-R^{\prime}\right)$ (see Figure B.4). Recall that $a$ is one of the intersection points of the circle centered at $\pi^{\prime}(p)$ of radius 1 and the circle centered at $O$ of radius $R+1-y^{\frac{2}{3}}$. A simple computation yields that the coordinates $\left(X_{a}, Y_{a}\right)$ of $a$ are equal to

$$
Y_{a}=\frac{\left(R+1-y^{\frac{2}{3}}\right)^{2}-1-R^{\prime 2}}{2 R^{\prime}}, \quad\left|X_{a}\right|=\sqrt{1-Y_{a}^{2}} .
$$

If $R^{\prime}=R$, then

$$
Y_{a}=\frac{y^{\frac{4}{3}}+2 R-2 R y^{\frac{2}{3}}-2 y^{\frac{2}{3}}}{2 R}=1-y^{\frac{2}{3}}\left(1+\frac{2-y^{\frac{2}{3}}}{2 R}\right) \geqslant 1-2 y^{\frac{2}{3}}
$$

which implies that

$$
\left|X_{a}\right| \leqslant \sqrt{1-\left(1-2 y^{\frac{2}{3}}\right)^{2}}=\sqrt{4 y^{\frac{2}{3}}-4 y^{\frac{4}{3}}} \leqslant 2 y^{\frac{1}{3}} .
$$

Now if $R^{\prime} \neq R$, then $\left(R+1-y^{\frac{2}{3}}\right)^{2}-1 \leqslant R^{2}$ by definition. Expanding this inequality yields

$$
\begin{gathered}
y^{\frac{4}{3}}+2 R-2 R y^{\frac{2}{3}}-2 y^{\frac{2}{3}} \leqslant 0, \\
y^{\frac{2}{3}} \geqslant \frac{y^{\frac{4}{3}}+2 R}{2(R+1)} \geqslant \frac{R}{R+1} \geqslant \frac{1}{2} .
\end{gathered}
$$

Thus $\sqrt{2 y^{\frac{2}{3}}} \geqslant 1$ and since $\left|X_{a}\right|=\sqrt{1-Y_{a}^{2}} \leqslant 1$ we get $\left|X_{a}\right| \leqslant \sqrt{2} y^{\frac{1}{3}}$. Hence, in both cases,

$$
\left|X_{a}\right| \leqslant 2 y^{\frac{1}{3}}
$$

Thus the length of the circular arc $a b$ is (see Figure B.5)

$$
\alpha=\left(R+1-y^{\frac{2}{3}}\right) \cdot 2 \arcsin \left(\frac{\left|X_{a}\right|}{R+1-y^{\frac{2}{3}}}\right) \leqslant\left(R+1-y^{\frac{2}{3}}\right) \cdot 2 \arcsin \left(\frac{2 y^{\frac{1}{3}}}{R+1-y^{\frac{2}{3}}}\right) .
$$




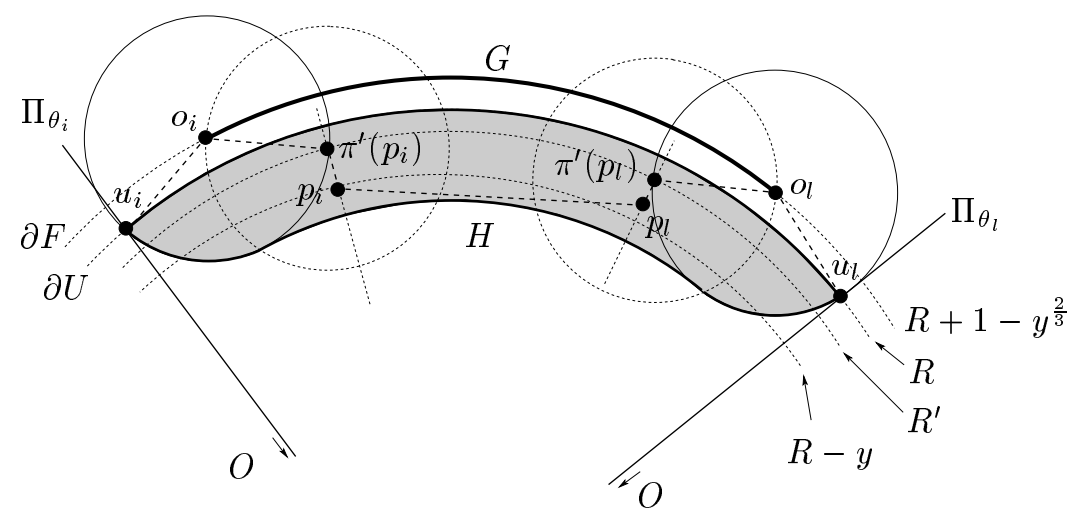

Fig. B.6. For the computing a bound on $\Delta \theta$.

A straightforward computation shows that $\arcsin (x)-\pi x \leqslant 0$ for any $x \in[0,1]$. Thus

$$
\alpha \leqslant\left(R+1-y^{\frac{2}{3}}\right) \cdot 2 \pi \frac{2 y^{\frac{1}{3}}}{R+1-y^{\frac{2}{3}}}=4 \pi y^{\frac{1}{3}} .
$$

Since the area $A$ of the middle part is less than or equal to $\alpha y^{\frac{2}{3}}$,

$$
A \leqslant 4 \pi y^{\frac{1}{3}} y^{\frac{2}{3}}=4 \pi y .
$$

This implies that the area of $\Pi \cap H$ is less than or equal to $12 \pi y$.

Lemma B.5. The volume of $H$ is bounded from above by $12 \pi^{2}(x+6) y$.

Proof. We express the volume of $H$ by an integral using spherical coordinates $(r, \theta, \phi)$ in an orthogonal frame $(O, \tilde{X}, \tilde{Y}, \tilde{Z})$ such that the plane $(O, \tilde{X}, \tilde{Y})$ contains $p_{i}$ and $p_{l}$ (see Figure B.3). A plane $\theta=$ constant contains the $\tilde{Z}$-axis and thus is a plane $\Pi$. Let $1_{H}(r, \theta, \phi)$ denote the indicator function of $H ; 1_{H}(r, \theta, \phi)$ is equal to 1 if the point of coordinates $(r, \theta, \phi)$ belongs to $H$ and to 0 otherwise. Then

$$
\text { Volume of } H=\int_{\phi} \int_{r} \int_{\theta} 1_{H}(r, \theta, \phi) \cdot r^{2} \sin \phi d r d \theta d \phi \text {. }
$$

Since $H$ is inside $\mathcal{U}, r \cdot 1_{H}(r, \theta, \phi) \leqslant R \cdot 1_{H}(r, \theta, \phi)$. Moreover $\sin \phi \leqslant 1$, thus

$$
\text { Volume of } H \leqslant R \int_{\theta}\left(\int_{\phi} \int_{r} 1_{H}(r, \theta, \phi) \cdot r d r d \phi\right) d \theta \text {. }
$$

The double integral in parentheses is equal to the area of the section of $H$ by a plane $\Pi_{\theta}: \theta=$ constant. By Lemma B.4, this area is less than $12 \pi y$, which is independent of $\theta$. Moreover the area is equal to 0 when $\Pi_{\theta}$ does not intersect $H$. Let $\Delta \theta$ denote the angle between the two extreme planes $\Pi_{\theta}$ that intersect $H$. Thus we have

$$
\text { Volume of } H \leqslant R \cdot 12 \pi y \cdot \Delta \theta \text {. }
$$

We now bound $\Delta \theta$. Refer to Figure B.6. Let $\Pi_{\theta_{i}}$ and $\Pi_{\theta_{l}}$ be the two extreme planes that intersect $H$. Let $u_{i}$ and $u_{l}$ be the two points of intersection of $H$ with $\Pi_{\theta_{i}}$ and $\Pi_{\theta_{l}}$, respectively; $u_{i}$ and $u_{l}$ lie on $\partial \mathcal{U}$. Let $o_{i}$ and $o_{l}$ be the two points in $G$ 
at distance 1 from $u_{i}$ and $u_{l}$, respectively. $\pi^{\prime}\left(p_{i}\right)$ and $\pi^{\prime}\left(p_{l}\right)$ are at distance 1 from $o_{i}$ and $o_{l}$, respectively.

The angle between the two extreme planes $\Pi_{\theta_{i}}$ and $\Pi_{\theta_{l}}$ is, as before,

$$
\Delta \theta=2 \arcsin \frac{\left|u_{i} u_{l}\right| / 2}{R} \leqslant 2 \pi \frac{\left|u_{i} u_{l}\right| / 2}{R}=\pi \frac{\left|u_{i} u_{l}\right|}{R} .
$$

Now we bound $\left|u_{i} u_{l}\right|$ by the length of the polygonal line shown in Figure B.6.

$$
\begin{aligned}
\left|u_{i} u_{l}\right| & \leqslant\left|u_{i} o_{i}\right|+\left|o_{i} \pi^{\prime}\left(p_{i}\right)\right|+\left|\pi^{\prime}\left(p_{i}\right) p_{i}\right|+\left|p_{i} p_{l}\right|+\left|p_{l} \pi^{\prime}\left(p_{l}\right)\right|+\left|\pi^{\prime}\left(p_{l}\right) o_{l}\right|+\left|o_{l} u_{l}\right| \\
& =1+1+\left|\pi^{\prime}\left(p_{i}\right) p_{i}\right|+x+\left|p_{l} \pi^{\prime}\left(p_{l}\right)\right|+1+1 .
\end{aligned}
$$

We show that $\left|\pi^{\prime}\left(p_{i}\right) p_{i}\right|$ and $\left|p_{l} \pi^{\prime}\left(p_{l}\right)\right|$ are less than $1 . \pi^{\prime}\left(p_{i}\right)$ is inside $\mathcal{U}$ at distance less than 1 from $\partial F$ which lies outside $\mathcal{U}$. Thus $\pi^{\prime}\left(p_{i}\right)$ is inside $\mathcal{U}$ at distance less than 1 from its frontier. Point $p_{i}$ is also inside $\mathcal{U}$ at distance less than 1 from its frontier. Since $p_{i}$ and $\pi^{\prime}\left(p_{i}\right)$ are on the same ray starting from $O$, they are at distance less than 1 apart. Similarly for $\pi^{\prime}\left(p_{l}\right)$ and $p_{l}$. Hence

$$
\Delta \theta \leqslant \pi \frac{\left|u_{i} u_{l}\right|}{R} \leqslant \pi \frac{x+6}{R}
$$

Therefore

$$
\text { Volume of } H \leqslant R \cdot 12 \pi y \cdot \Delta \theta \leqslant 12 \pi^{2} y(x+6) \text {. }
$$

Proposition B.1 follows from Lemmas B.3 and B.5.

Appendix C. Volume of the intersection of two spherical shells. We prove in this section the following proposition used in the proof of Lemma 3.17.

Proposition C.1. Let $R>0, x \in[6,2 R], y \in[0,1]$ and $p$ be a point at distance $R-y$ from $O$. The volume of the intersection of the region in between the two spheres centered at $p$ and of radii $x$ and $x+d x$, and the region in between the two spheres centered at $O$ and of radii $R$ and $R-y$ (see Figure C.1) is bounded from above by $8 \pi x y d x$.

Proof. Define the balls $B_{1}$ with center $O$ and radius $R, B_{2}$ with center $O$ and radius $R-y, B_{3}$ with center $p$ and radius $x$ and finally $B_{4}$ with center $p$ and radius $x+d x$. Let $\mathcal{V}$ denote the intersection of $\left(B_{1} \backslash B_{2}\right)$ and $\left(B_{4} \backslash B_{3}\right)$. We prove that the volume of $\mathcal{V}$ is less than $8 \pi x y d x$.

Since $d x$ is infinitesimally small, the volume of $\mathcal{V}$ is $\mathcal{A} d x$ where $\mathcal{A}$ is the area of the intersection of the sphere $\partial B_{3}$ with $B_{1} \backslash B_{2}$.

Let $(p, X, Y, Z)$ be an orthogonal reference frame whose center is $p$ and whose $X$ axis is oriented along $\overrightarrow{O p}$ (see Figure C.1). Notice that all spheres are centered on that axis. Let $C_{1}$ (resp. $C_{2}, C_{3}$ ) denote the circle that is the boundary of the intersection of $B_{1}$ (resp. $\left.B_{2}, B_{3}\right)$ and the plane $(p, X, Y)$ in which Figure C. 1 is drawn. The equations of these circles are, in the frame $(p, X, Y)$,

$$
\begin{aligned}
& C_{1}:(X+R-y)^{2}+Y^{2}=R^{2}, \\
& C_{2}:(X+R-y)^{2}+Y^{2}=(R-y)^{2} \text {, } \\
& C_{3}: \quad X^{2}+Y^{2}=x^{2} \text {. }
\end{aligned}
$$

Since $C_{3}$ is centered at a point on $C_{2}$ and has radius $x \geqslant 6>1 \geqslant y, C_{3}$ intersects or encloses $C_{1}$ and $C_{2}$. In fact, $C_{3}$ intersects or encloses $C_{1}$ and $C_{2}$ in one of the three following ways. 


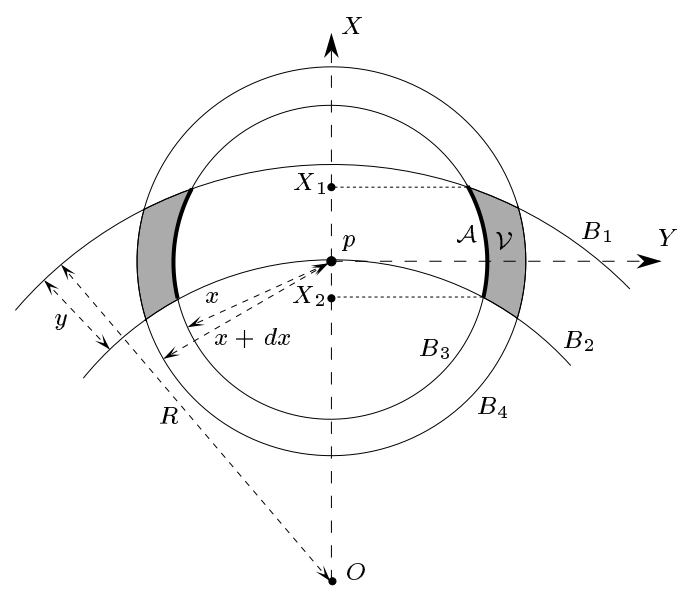

(a)

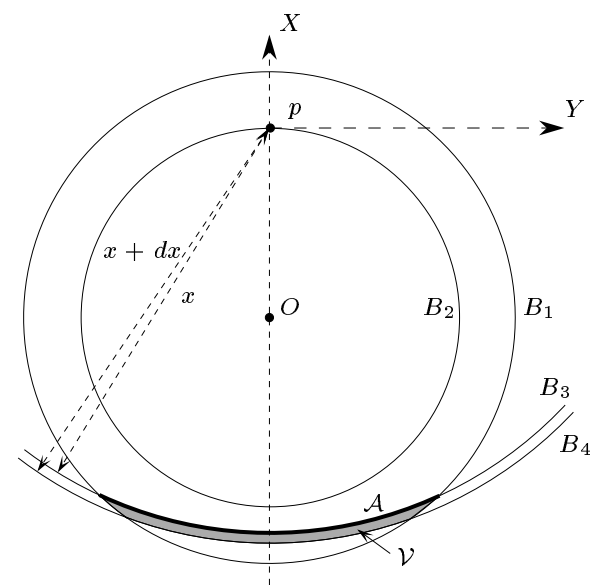

(b)

Fig. C.1. For the proof of Proposition C.1.

Case 1: If $6 \leqslant x \leqslant 2 R-2 y$ then $C_{3}$ intersects both $C_{1}$ and $C_{2}$ (see Figure C.1 (a)). Case 2: If $2 R-2 y<x \leqslant 2 R-y$ then $C_{3}$ intersects $C_{1}$ and encloses $C_{2}$ (see Figure C.1 (b)).

Case 3: If $2 R-y<x$ then $C_{3}$ encloses both $C_{1}$ and $C_{2}$. In that case, $\mathcal{V}$ is empty and the volume is 0 .

In the first case, let $X_{1}$ (resp. $X_{2}$ ) be the abcissa of the points of intersection of circles $C_{1}$ (resp. $C_{2}$ ) and $C_{3}$. Note that $X_{1} \geqslant X_{2}$ and their values can be computed directly from the equations of the circles $C_{1}, C_{2}$ and $C_{3}$ :

$$
X_{1}=\frac{R^{2}-x^{2}-(R-y)^{2}}{2 R-2 y}, \quad X_{2}=\frac{-x^{2}}{2 R-2 y} .
$$

Using the fact that $y \leqslant x \leqslant 2 R-2 y$ we get

$$
\begin{aligned}
X_{1}-X_{2} & =\frac{y(2 R-y)}{2 R-2 y}=y\left(1+\frac{y}{2 R-2 y}\right) \leqslant 2 y, \\
-X_{1}-X_{2} & =\frac{2 x^{2}-y(2 R-y)}{2 R-2 y} \leqslant 2 x \frac{x}{2 R-2 y} \leqslant 2 x .
\end{aligned}
$$

We now bound from above the area $\mathcal{A}$ of the surface $\partial B_{3} \cap\left(B_{1} \backslash B_{2}\right)$ by the area of a larger surface which depends on the sign of $X_{1}$. If $X_{1} \geqslant 0$, the surface consists of a cylinder of axis the $X$-axis, of radius $x$ and height $X_{1}-X_{2}$, and of two annuli in the planes $X=X_{1}$ and $X=X_{2}$, of inner radius $\sqrt{x^{2}-X_{1}^{2}}$ and $\sqrt{x^{2}-X_{2}^{2}}$, respectively, and outer radius $x$ (see Figure C.2 (a)). If $X_{1} \leqslant 0$, the surface consists of a cylinder of axis the $X$-axis, of radius $\sqrt{x^{2}-X_{1}^{2}}$ and height $X_{1}-X_{2}$, and of an annulus in the plane $X=X_{2}$, of inner radius $\sqrt{x^{2}-X_{2}^{2}}$ and outer radius $\sqrt{x^{2}-X_{1}^{2}}$ (see Figure C.2 (b)). In both cases that surface is larger than $\partial B_{3} \cap\left(B_{1} \backslash B_{2}\right)$ by convexity.

If $X_{1} \geqslant 0$, the area of the cylinder is $2 \pi x\left(X_{1}-X_{2}\right) \leqslant 4 \pi x y$ and the area of the annuli are $\pi x^{2}-\pi\left(x^{2}-X_{i}^{2}\right)=\pi X_{i}^{2}, i=1,2$. Since $X_{1} \geqslant 0, X_{1} \leqslant y \leqslant x$ and thus $\pi X_{1}^{2} \leqslant \pi x y$. We also have from the expression of $X_{1}$ that $R^{2}-x^{2}-(R-y)^{2} \geqslant 0$ and thus $x^{2} \leqslant y(2 R-y)$. Thus

$$
\pi X_{2}^{2}=\pi \frac{x^{2}}{2 R-2 y} \frac{x^{2}}{2 R-2 y} \leqslant \pi x \frac{x}{2 R-2 y} y \frac{2 R-y}{2 R-2 y}=\pi x y \frac{x}{2 R-2 y}\left(1+\frac{y}{2 R-2 y}\right) .
$$




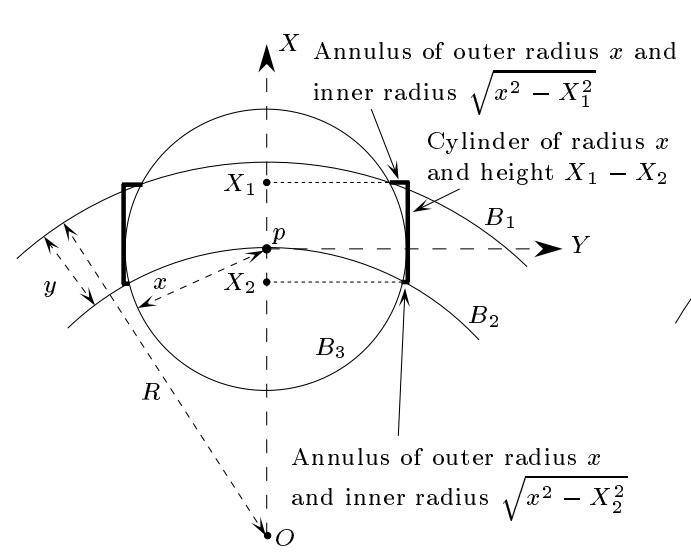

(a)

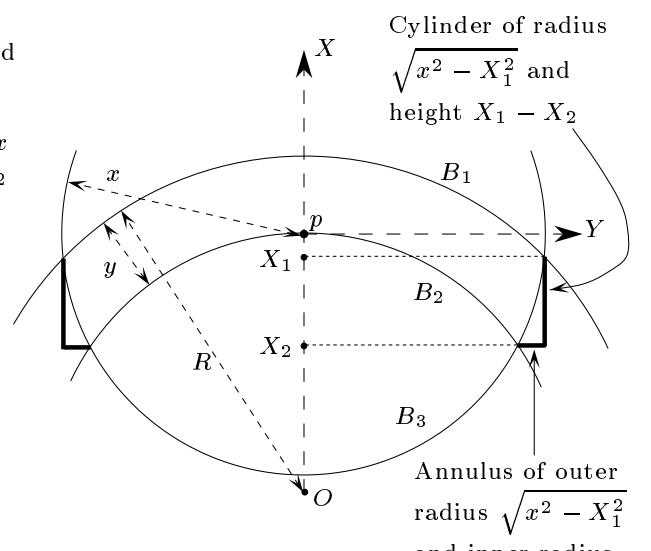

(b) and inner radius $\sqrt{x^{2}-X_{2}^{2}}$

Fig. C.2. For the proof of Proposition C.1, case 1.

It thus follows from $y \leqslant x \leqslant 2 R-2 y$ that $\pi X_{2}^{2} \leqslant 2 \pi x y$. Hence $\mathcal{A} \leqslant 7 \pi x y$.

If $X_{1} \leqslant 0$, the area of the cylinder is $2 \pi \sqrt{x^{2}-X_{1}^{2}}\left(X_{1}-X_{2}\right) \leqslant 2 \pi x(2 y)$ and the area of the annulus is $\pi\left(x^{2}-X_{1}^{2}\right)-\pi\left(x^{2}-X_{2}^{2}\right)=\pi\left(X_{1}-X_{2}\right)\left(-X_{2}-X_{1}\right) \leqslant 4 \pi x y$. Thus $\mathcal{A} \leqslant 8 \pi x y$.

Consider now the second case $2 R-2 y<x \leqslant 2 R-y$ (see Figure C.1 (b)). For a fixed value of $y, \mathcal{A}$ is the area of a spherical cap whose perimeter and curvature decreases as $x$ increases. Thus $\mathcal{A}$ is a decreasing function of $x$. Since the bound $\mathcal{A} \leqslant 8 \pi x y$ is valid for $x=2 R-2 y$ and $8 \pi x y$ is an increasing function of $x, \mathcal{A} \leqslant 8 \pi x y$ for any $x \geqslant 2 R-2 y$.

Acknowledgments. The authors would like to thank Helmut Alt who led us to the lower bound proof of Section 4 and Luc Devroye for useful discussions.

\section{REFERENCES}

[1] P. K. Agarwal, B. Aronov, AND M. Sharir, Line transversals of balls and smallest enclosing cylinders in three dimensions, Discrete and Computational Geometry, 21 (1999), pp. 373388 .

[2] B. Aronov, H. Brönnimann, A. Chang, And Y.-J. Chiang, Cost prediction for ray shooting, in Proceedings of the ACM Symposium on Computational Geometry, Barcelona, Spain, 2002, pp. 293-302.

[3] M. DE BERG, H. Everett, AND L.J. Guibas, The union of moving polygonal pseudodiscs Combinatorial bounds and applications, Computational Geometry: Theory and Applications, 11 (1998), pp. 69-82.

[4] F. S. CHO AND D. FORSYTH, Interactive ray tracing with the visibility complex, Computers and Graphics, Special Issue on Visibility - Techniques and Applications, 23:5 (1999), pp. $703-717$.

[5] O. Devillers, B. Mourrain, F. P. Preparata, and P. Trebuchet, On circular cylinders by four or five points in space, Rapport de recherche 4195, INRIA, Le Chesnay Cedex, France, 2001.

[6] O. Devillers and P. Ramos, Personal communication, 2001.

[7] F. Duguet and G. Drettakis, Robust epsilon visibility, ACM Transactions on Graphics, 21:3 (2002), pp. 567-575. Proc. Siggraph'02.

[8] F. DuRAND, A multidisciplinary survey of visibility, ACM Siggraph course notes, Visibility, Problems, Techniques, and Applications, 2000. 
[9] F. Durand, G. Drettakis, and C. Puech, The visibility skeleton: a powerful and efficient multi-purpose global visibility tool, In Computer Graphics Proc. Ann. Conference Series, 31 (1997), pp. 89-100. Proc. Siggraph'97.

[10] F. Durand, G. Drettakis, and C. Puech, Fast and accurate hierarchical radiosity using global visibility, ACM Transactions on Graphics, 18:2 (1999), pp. 128-170.

[11] F. Durand, G. Drettakis, and C. Puech, The 3D visibility complex, ACM Transactions on Graphics, 21:2 (2002), pp. 176-206.

[12] G. R. Grimmett and D. R. Stirzaker, Probability and Random Processes (2nd edition), Clarendon Press - Oxford, Oxford, 1992.

[13] N. Holzschuch, F. Sillion, and G. Drettakis, An efficient progressive refinement strategy for hierarchical radiosity, In Proceedings of the 5th Eurographics Workshop on Rendering, pp. 353-357, 1994, G. Sakas, P. Shirley and S. Muller (editors), Photorealistic Rendering Techniques, Springer, Focus on Computer Graphics Series, Berlin, 1995.

[14] A. LAURENTINI, The visual hull concept for silhouette-based image understanding, IEEE Transactions on Pattern Analysis and Machine Intelligence, 16:2 (1994), pp. 150-162.

[15] I.G. Macdonald, J. Pach, and T. Theobald, Common tangents to four unit balls in $\mathbb{R}^{3}$, Discrete and Computational Geometry 26:1 (2001), pp. 1-17.

[16] The Maple System. Waterloo Maple Software. http://www.maplesoft.com/.

[17] A. M. Mathai, An Introduction to Geometrical Probability: Distributional Aspects with Applications, Gordon and Breach Sciences Publishers, 1999.

[18] B. Nadler, G. Fibich, S. Lev-Yehudi, And D. Cohen-Or, A qualitative and quantitative visibility analysis in urban scenes, Computers and Graphics 23:5 (1999), pp. 655-666.

[19] Athanasios Papoulis, Probability, Random Variables, and Stochastic Processes, 3rd ed. McGraw-Hill, 1991.

[20] M. Pellegrini, Ray shooting on triangles in 3-space, Algorithmica, 9 (1993), pp. 471-494.

[21] S. Petitjean, The number of views of piecewise-smooth algebraic objects, In Proceedings of the Sympos. Theoret. Aspects of Comput. Sci., Lecture Notes in Computer Science, 900 (1995), pp. 571-582.

[22] H. Plantinga And C. R. Dyer, Visibility, occlusion, and the aspect graph, Internat. J. Comput. Vision, 5:2 (1990), pp. 137-160.

[23] M. Pocchiola and G. Vegter, The visibility complex, Internat. J. Comput. Geom. Appl., 6:3 (1996), pp. $279-308$

[24] L.A. Santaló, Integral Geometry and Geometric Probability, Addison-Wesley Pub. 1976.

[25] L. Szirmay-Kalos, V. Havran, B. Balazs, and L. Szecsi, On the efficiency of ray-shooting acceleration schemes, in Proceedings of the Spring Conference on Computer Graphics, Budmerice, Slovakia, 2002, pp. 89-98.

[26] L. Szirmay-Kalos And G. MÁRTOn, Worst-case versus average case complexity of rayshooting, Computing, 61:2 (1998), pp. 103-131. 